\title{
Bundling Electronic Journals and Competition among Publishers*
}

\author{
Doh-Shin Jeon $^{\dagger}$ and Domenico Menicucci ${ }^{\ddagger}$ \\ This version: October 20, 2005, the first version: April 21, 2003
}

\begin{abstract}
Site licensing of electronic journals has been revolutionizing the way academic information is distributed. However, many librarians are concerned about the possibility that commercial publishers might abuse site licensing by practicing bundling. In this paper, we analyze how bundling affects journal pricing in the market of scientific, technical and medical (STM) electronic journals and offer a novel insight on the bundling of a large number of information goods. We find that (i) when bundling is prohibited, surprisingly, industry concentration does not affect prices (ii) when bundling is allowed, each publisher finds bundling profitable and bundling increases industry profits while reducing social welfare and (iii) any merger among publishers already active in the market is profitable but reduces social welfare.
\end{abstract}

Keywords: Bundling, Journal Pricing, Site Licensing, Mergers

JEL numbers: D4, K21, L41, L82

\footnotetext{
${ }^{*}$ We thank seminar participants at University of Bristol, University of Malaga, University of Montpellier, Universitat Pompeu Fabra, EARIE 2004, ESEM-EEA 2003, IIOC 2004 and Toulouse conference on the economics of the software and Internet industries 2005. We are grateful to Roberto Burguet, Antonio Cabrales, Patrick Rey, Jean-Charles Rochet, Joel Shapiro, Jean Tirole and an anonymous referee for useful comments. The first author gratefully acknowledges the financial support from the Spanish Ministry of Science and Technology under the Ramon y Cajal grant and from DGES and FEDER under BEC2003-00412.

†Universitat Pompeu Fabra and CREA, Barcelona, Spain. doh-shin.jeon@upf.edu

${ }^{\ddagger}$ Università di Firenze, Italy. domenico.menicucci@dmd.unifi.it
} 


\section{Introduction}

Site licensing of electronic journals (e-journals, henceforth) has been revolutionizing the way academic information is distributed. Under site licensing, there is no need to spend time to look for a paper in a library and many people can download, read and print a paper simultaneously from their offices at any given time. Furthermore, e-journals' websites provide additional services such as search tools, hypertext linking, remote access etc. Therefore it seems that, sooner or later, e-journals will supplant print journals as the norm.

However, many librarians are concerned about the possibility that commercial publishers might abuse site licensing for private gain. First, commercial publishers have aggressively raised prices at a rate disproportionate to any increase in costs or quality. According to the Association of Research Libraries (ARL) in the U.S., ${ }^{1}$ during the period of 1986-2002, the unit cost for journal subscriptions has grown at the rate of $7.7 \%$ per year, which is more than twice the growth rate of the unit cost of monographs, $3.6 \%$. As Figure 1 shows, up to 2000, the increase in the budget of the libraries could not match the increase in journal prices, which resulted in a continuous decrease in the amount of journals purchased during most of the period. High subscription prices charged by commercial publishers even induced some academic societies whose journals had been published by commercial publishers to start new competing journals, as in the case of the launch of Journal of the European Economic Association by European Economic Association. ${ }^{2}$ Second, site licensing of e-journals allows commercial publishers to employ powerful pricing strategies such as price discrimination based on usage ${ }^{3}$ and bundling while, with print journals, they practiced neither bundling nor discrimination between libraries in terms of subscription prices. ${ }^{4}$ In particular, librarians are concerned about bundling. For instance, according to Kenneth Frazier (2001), director of libraries at University of WisconsinMadison, "the content is 'bundled' so that individual journal subscriptions can no longer

\footnotetext{
${ }^{1}$ See "Monograph and Serial Costs in ARL Libraries 1986-2002" at http://www.arl.org/stats/arlstat/.

${ }^{2}$ In other disciplines, there are several cases in which the editorial board of a journal owned by a commercial publisher resigned and founded an alternative journal. See Theodore Bergman's website: http://www.econ.ucsb.edu/ tedb/Journals/alternatives.html

${ }^{3}$ For instance, Derk Haank (2001), the CEO of Elsevier Science, says "What we are basically doing is to say that you pay depending on how useful the publication is for you - estimated by how often you use it." See also Bolman (2002) and Key Perspectives (2002) about price discrimination.

${ }^{4}$ In the case of print journals, arbitrage through resale has to some extent limited publishers' ability to practice price discrimination. In contrast, in the case of e-journals, access to a journal is simply leased and hence resale is impossible.
} 


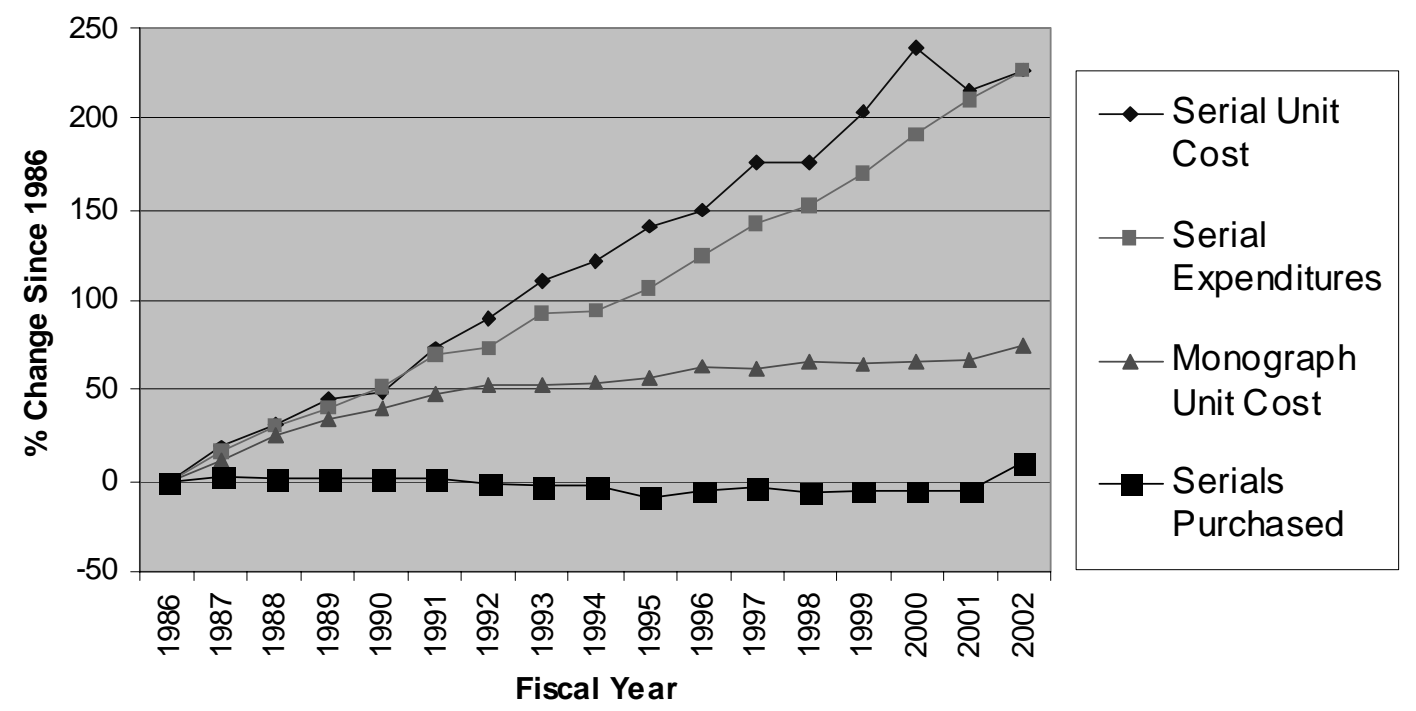

Figure 1: Monograph and serial costs in ARL libraries, 1986-2002 (source: ARL)

be cancelled in their electronic format. (The Academic Press IDEAL program and the full ScienceDirect package offered by Elsevier are examples of such licensing agreements)." 5

Moreover, U.S. and U.K. competition authorities approved four years ago one of the biggest-ever science publishing mergers between Reed-Elsevier (RE henceforth) and Harcourt in spite of many librarians' protests. Indeed, the report of U.K. Competition Commission (2001) shows concern about potential welfare losses due to the merging publishers' bundling of their e-journals. Before the merger, RE's ScienceDirect was the most developed website and offered access to around 1,150 journals and Harcourt's IDEAL offered access to 320 journals.

In this paper, we analyze publishers' incentives to practice bundling, the ensuing effects on social welfare and derive implications for merger analysis. In order to isolate the effect of bundling under price discrimination, we consider a mature stage of e-journals in which publishers practice price discrimination based on usage. ${ }^{6}$ Therefore, we assume away

\footnotetext{
${ }^{5}$ He further argues that "the push to build an all-electronic collection can't be undertaken at the risk of: (1) weakening that collection with journals we neither need nor want, and (2) increasing our dependence on publishers who have already shown their determination to monopolize the information market place."

${ }^{6}$ This implies that the pricing schemes we study in this paper might not correspond to what we observe now. In fact, the transition implies a change from subscription-based pricing models to usagebased models and since a sudden switch in the pricing models generates a large change in the total
} 
heterogeneity among libraries and build a model in which each competing publisher offers a set of journals to a library which wants to build a portfolio of journals and monographs under a budget constraint. ${ }^{7}$ We analyze how bundling affects journal pricing through its impact on the library's allocation of budget between journals and books. Although we assume that there is no direct substitution among journals in that the value the library derives from a journal is determined independently of whether or not it buys any other journal, there can be an indirect substitution among journals and among journals and monographs ${ }^{8}$ through the budget constraint. The utility that the library derives from spending money on books is assumed to be strictly increasing and strictly concave.

We first consider independent pricing (i.e. no-bundling) and show an irrelevance result in that industry concentration does not affect prices. For instance, in the simple case of homogeneous journals (in which every journal has the same value), we show that regardless of the level of industry concentration, there exists a unique equilibrium in which all the journals are sold at the same price. In the general case of heterogeneous journals, we show that there is a unique equilibrium candidate regardless of the level of industry concentration and that the equilibrium always exists both under the maximum concentration (i.e. the monopoly case) and under the minimum concentration in which each publisher sells only one journal. ${ }^{9}$ Therefore, the outcome under the minimum concentration is equivalent to the outcome under the maximum concentration. The irrelevance result is related to the fact that under independent pricing, each journal is priced according to what we call "marginal opportunity cost pricing" in the following sense: when a publisher sells a journal, he expects that his journal is the marginal journal (i.e. the last journal purchased by the library) and chooses a price $p$ to match the library's opportunity cost of using $p$ instead on books, such that the library is indifferent between buying the journal at $p$ and spending $p$ on buying extra books. A monopolist cannot realize a higher profit than the one under marginal opportunity cost pricing since, in order to realize a higher profit, he has to increase the price of the marginal journal and this induces the library not to buy the journal.

price that allows a library to maintain its subscription to a given collection of journals, publishers are introducing a progressive change (Bolman, 2002).

${ }^{7}$ Typically, an academic library's material budget is spent on journals and monographs (Gooden et al., 2002).

${ }^{8}$ Because of journal price increases, many university libraries have been forced to reallocate dollars from monographs to journals (Kyrillidou, 1999).

${ }^{9}$ For the intermediate case of oligopoly, we give a sufficient condition for equilibrium existence in theorem 2 in section 4 . The irrelevance result holds as long as the equilibrium exists. 
When bundling is allowed, we show that each publisher has an incentive to bundle all his journals. We identify two effects of bundling. First, bundling has the direct effect of softening competition from books. To provide intuition, we consider a monopolist who publishes two journals of the same value $u$. Under independent pricing, he expects that each of his journals is the last journal to be purchased and chooses the same price $p$ for them. Suppose now that he bundles the journals and chooses $2 p$ as a price for the bundle. Then, the library is strictly better off by buying the bundle than by spending $2 p$ on books; since the marginal utility from spending money on books strictly decreases, the utility from spending $2 p$ on books is strictly smaller than twice the utility from spending only $p$ on books. Therefore, the monopolist can charge $2 p+\varepsilon(>2 p)$ for the bundle and still induce the library to buy it. This direct effect of bundling increases with the size of bundle, which implies that a large publisher gains more than a small publisher in terms of the direct effect.

Second, a publisher's bundling has an indirect effect of inflicting negative pecuniary externalities on all the other publishers. The very fact that bundling allows a publisher to increase his profit implies that after a publisher's bundling, there is less budget left for books and all the other publishers' journals. This in turn implies that for all the other publishers, competition from books is tougher and therefore they have to lower the prices of their journals in order to sell them. In particular, a small publisher which has only a few journals does not gain much from the direct effect of bundling, but may lose a lot from the indirect effect if big publishers bundle their journals. Therefore, bundling is a profitable and credible strategy: it not only increases the bundling publisher's profit but also decreases the profits of rivals and can even make them unable to sell their journals.

The direct and indirect effects of bundling suggest that any merger increases the merging publishers' profits because of the direct effect while reducing rivals' profits because of the indirect effect. We also show that bundling (or any merger) increases industry profits. This result implies that the library consumes less books after bundling. Since bundling can make small publishers unable to sell their journals, we conclude that bundling decreases social welfare by reducing both book and journal consumption. For the same reasons, any merger among active publishers reduces social welfare. Our finding is consistent with the prediction of Kyrillidou (1999) that if the current trend continues, the budget for monographs will be the resource depleted fastest, and that only about $10 \%$ of the materials budget will be spent on purchasing monographs by 2019. Finally, when we examine publishers' incentive to acquire a journal from a third-party, we find that in the absence of bundling all the publishers have the same willingness to pay for the journal, while under 
bundling, the largest publisher has always the highest willingness to pay. This suggests that bundling might seriously affect industry dynamics such that the largest publisher becomes even larger through the purchase of the titles sold by small publishers forced to exit the market.

Most of the papers on bundling study bundling of two (physical) goods in the context of second-degree price discrimination and focus on either surplus extraction (Schmalensee, 1984, McAfee et al. 1989, Salinger 1995 and Armstrong 1996, 1999) or entry deterrence (Whinston 1990 and Nalebuff 2004). Bakos and Brynjolfsson (1999, 2000)'s papers are an exception in that they study bundling of a large number of information goods while maintaining the second-degree price discrimination framework. Their first paper shows that bundling allows a monopolist to extract more surplus since it reduces the variance of average valuations by the law of large numbers $;{ }^{10}$ the second paper applies this insight to entry deterrence. Our paper also studies bundling of any number of information goods. The novelty is that we show that bundling is a profitable and credible strategy both in terms of surplus extraction and entry deterrence even when sellers have complete information on the buyer's valuation for each product (and therefore the law of large numbers plays no role) and there is no interdependency among valuations of different products. Conventional wisdom says that bundling has no effect in such a setting and this is true if the budget constraint is not binding. However, when the constraint is binding, we show that each firm has a strict incentive to adopt bundling.

Our paper is related to McCabe (2002b), who studies the transition from print journals (no price discrimination and no bundling) to e-journals (prefect price discrimination and bundling). ${ }^{11}$ Although his setting is similar to ours, there are important differences. First, he considers the transition while we consider the situation when this transition is over. Second, he does not provide the comparative statics of the transition while we provide the comparative statics of bundling versus no-bundling in the digital world. Furthermore, he assumes bundling in the case of e-journals while we show that in equilibrium all publishers adopt bundling. Lastly, he does not consider the substitution between books and journals and his results often rely on numerical examples. Edlin and Rubinfeld (2004) also argue that bundling of academic journals builds strategic barriers to entry but do not build any formal model. McCabe and Snyder (2004) analyze the market for academic journals from a two-sided market perspective but do not study bundling.

\footnotetext{
${ }^{10}$ See also Armstrong (1999).

${ }^{11}$ McCabe (2002a) provides an empirical analysis showing that mergers significantly contributed to journal price increases.
} 
Finally, the comparison between bundling and independent pricing in our paper is related to the comparison between patent pooling and independent licensing of patents in Lerner and Tirole (2004). In particular, publishers' pricing decisions in our paper are driven by what they call the competitive margin in that each publisher cannot raise his prices without triggering an exclusion of his journal(s) from the portfolio of journals bought by the library. However, there are two important differences. First, they consider a simple case in which each owner owns only one patent and hence there is no issue of patent pooling at the individual owner level while we consider a general case in which each firm owns multiple journals and therefore bundling is decided at the firm level. Second, as a consequence, patent pooling implies a change from the minimum industry concentration to a monopoly in their paper while we compare bundling with independent pricing for any given level of industry concentration.

The rest of the paper is organized as follows. Section 2 describes the model. In Section 3, we consider the simple case of homogeneous journals and explain all our main results with minimum technical details. In Section 4, we consider the general case of heterogeneous journals. Section 5 provides concluding remarks. All the proofs which do not appear in the main text are relegated to Appendix.

\section{Model}

As we said in the introduction, we consider the mature stage of site-licensing in which journal prices depend on usage and assume that publishers have complete information about the value that a library attaches to a journal. This assumption allows us to focus on the effects of bundling which arise when buyers have no private information. Therefore, we consider only one library with a budget $M(>0)^{12}$ that is assumed to be known to all publishers.

\subsection{Journals and publishers}

There are $N$ publishers; publisher $j$ is often denoted simply by $j$. We only consider profitmaximizing publishers. Let $n_{j} \geq 1$ be the number of journals that publisher $j$ publishes $(j=1, \ldots, N)$ and $n \equiv \sum_{j=1}^{N} n_{j}(\geq N)$ the total number of journals. Let $u_{i j}>0$ represent the utility (or the surplus) the library obtains from journal $i=1, \ldots, n_{j}$ of publisher $j$. Let

\footnotetext{
${ }^{12}$ Considering only one library is without loss of generality in our framework since publishers can price-discriminate with respect to $u_{i j}$ and $M$.
} 
$U_{j} \equiv \sum_{i=1}^{n_{j}} u_{i j}$ and $U \equiv \sum_{j=1}^{N} U_{j}$. Journals are said to be homogeneous when $u_{i j}=u>0$ for all $i$ and $j$.

Since we focus on how bundling affects which journals are sold and at what prices, we assume that $n$ number of journals are already produced (i.e. the fixed cost of having the first copy of each journal has already been incurred). We assume that the marginal cost of distributing a journal is zero.

When each journal is sold independently (i.e. in the absence of bundling), publisher $j$ chooses price $p_{i j}>0$ for each journal $i$ he owns. Let $\mathbf{p} \equiv\left(p_{11}, \ldots, p_{n_{1} 1}, \ldots, p_{1 N}, \ldots, p_{n_{N} N}\right) \in$ $\mathbb{R}_{++}^{n}$ represent the price vector under independent pricing. Under bundling, publisher $j$ chooses price $P_{j}>0$ for the bundle of all his journals, which we denote by $\mathrm{B} j$. Let $\mathbf{P} \equiv\left(P_{1}, \ldots, P_{N}\right) \in \mathbb{R}_{++}^{N}$ denote the price vector under bundling.

\subsection{The library}

The library's budget $M(>0)$ is given and we study how bundling affects the library's allocation of the budget between journals and books (monographs). The library's payoff is given by the sum of three components: the utility it obtains from the journals it purchased, the utility it obtains from the books it bought and the money left after the purchases. We define a reduced-form utility for books by using an indirect utility function $v:[0,+\infty) \rightarrow \mathbb{R}_{+}$such that $v(m)$ is the library's utility from books when it spends $m \geq 0$ amount of money on buying books. $v(m)$ satisfies $v(0)=0$ and $v^{\prime}(m)>0>v^{\prime \prime}(m)$ for any $m \geq 0$. We further assume that $v^{\prime}(m)>1$ for all $m \leq M$; therefore the library prefers buying books to keeping money.

When each journal is sold independently, we let $x_{i j} \in\{0,1\}$ represent the library's choice about journal $i j: x_{i j}=1\left(x_{i j}=0\right)$ means that the library buys (does not buy) this journal. When all publishers use bundling, $X_{j} \in\{0,1\}$ represents the library's choice about $\mathrm{B} j: X_{j}=1\left(X_{j}=0\right)$ means that the library buys (does not buy) this bundle. Let $\mathbf{x} \equiv\left(x_{11}, \ldots, x_{n_{1} 1}, \ldots, x_{1 N}, \ldots, x_{n_{N} N}\right) \in\{0,1\}^{n}$ and $\mathbf{X} \equiv\left(X_{1}, \ldots, X_{N}\right) \in\{0,1\}^{N}$. Under independent pricing, given $(\mathbf{p}, M)$, the library chooses $\mathbf{x}$ and $m(\geq 0)$ to maximize its payoff ${ }^{13}$

$$
\sum_{j=1}^{N} \sum_{i=1}^{n_{j}} u_{i j} x_{i j}+v(m)+\left[M-\sum_{j=1}^{N} \sum_{i=1}^{n_{j}} p_{i j} x_{i j}-m\right]
$$

\footnotetext{
${ }^{13}$ As a tie-breaking rule, we assume that (i) if the library is indifferent between buying a journal (or a bundle) and not buying it, it buys the journal/bundle and (ii) if it is indifferent between two or more combinations of journals and/or bundles, it chooses the combination with the highest aggregate value of journals.
} 
subject to the budget constraint $\sum_{j=1}^{N} \sum_{i=1}^{n_{j}} p_{i j} x_{i j}+m \leq M .{ }^{14}$ The library's maximization problem under bundling is similarly defined using $\left(U_{1}, \ldots, U_{N}\right), \mathbf{P}$ and $\mathbf{X}$.

\subsection{Social welfare}

Social welfare is defined as the sum of the payoff of the library, the profits of the journal publishers and the profit of the book industry. The cost of producing a book is composed of a fixed cost and a marginal cost, about which we make a simplifying assumption: the fixed cost incurred by the book industry is not affected by the library's choice of $m$ and the marginal cost of producing a book is zero. ${ }^{15}$ Then, social welfare is equal to the total utility the library obtains from journals and books up to a constant.

\subsection{Timing and equilibrium selection}

We consider the following game, denoted by $\Gamma$, in which each publisher simultaneously decides (i) whether to be active or not and, if active, decides (ii) whether to bundle or not his journals and (iii) the price(s) of his bundle or journals. If a publisher is not active, he does not offer any journal or bundle and therefore the library cannot buy his journal(s) or bundle.

For equilibrium selection, we assume in section 3, in which we consider homogeneous journals, that a publisher decides not to be active if he expects to make zero profit (i.e. if he expects that the library will not buy his bundle or any of his journals). The assumption can be justified if a publisher should incur a very small but positive cost of contracting with the library. Without this assumption, the prices of some items (journals/bundles) the library buys may depend on the prices of the items the library does not buy, as we show through an example in subsection 3.2.

We first study in subsection 3.1 the game in which no publisher bundles his journals; we use $\Gamma^{I}$ to denote this game. Then, in subsection 3.2, we analyze the game in which all active publishers bundle their journals, denoted by $\Gamma^{B}$, and in subsection 3.3 we examine each publisher's incentive to choose between bundling and no-bundling in game $\Gamma$.

\footnotetext{
${ }^{14}$ In subsection 2.4 we describe how each publisher should first decide whether or not he will be active before choosing prices. Hence, (1) is correct if all publishers become active. If some are not active, $j$ runs over the set of active publishers.

${ }^{15}$ This is only a simplifying assumption. Our social welfare analysis is not qualitatively affected when the cost incurred by the book industry, denoted by $c(m)$, depends on $m$ as long as consuming more books is desirable from social point of view (i.e. $v^{\prime}(m)-c^{\prime}(m)>0$ for $m \leq M$.)
} 


\section{The simple case of homogeneous journals}

In this section we derive all our main results in the simple case of homogeneous journals, which means that $u_{i j}=u>0$ for all $i j$.

\subsection{Independent pricing}

We begin our analysis by examining $\Gamma^{I}$, the game in which each active publisher prices his journals independently. It turns out that in equilibrium the competitive margin binds (Lerner and Tirole, 2004) in that each publisher cannot raise the price of any of his journals without inducing the library to exclude the journal from the portfolio of journals it buys. For expositional facility, we first introduce the concept of a marginal bundle of books as follows. Consider the decision problem that the library faces with respect to the marginal journal (i.e. the last journal it purchases) with price $p$. If the library does not buy this journal, it can use $p$ to buy extra books. Let $\pi(\leq M)$ denote the journal industry profit when the marginal journal is bought. We define the marginal bundle of books corresponding to price $p$ as all the books that the library wishes to buy with $p$ after already spending $M-\pi$ on books. Then, the utility from the marginal bundle of books is given by

$$
U_{M B}(p, \pi) \equiv v(M-\pi+p)-v(M-\pi)>0 .
$$

Hence, when $u=U_{M B}(p, \pi)$ holds, the publisher selling the marginal journal cannot raise its price without triggering an exclusion of the journal from the list of the journals bought by the library. We describe in next lemma some properties of $U_{M B}$ which will be frequently used in the rest of the paper.

Lemma 1 (i) $U_{M B}$ strictly increases both with $p$ and with $\pi$.

(ii) $U_{M B}$ is strictly concave in $p$.

The proof of the lemma is omitted since it follows directly from the fact that $v(\cdot)$ is strictly increasing and strictly concave.

As a benchmark, we consider the case of minimum industry concentration in which each publisher owns only one journal $(N=n)$. Suppose that all publishers charge the same price $p\left(\leq \frac{M}{n}\right)$. Then, the library prefers buying $n^{\prime}$ number of journals (with $1 \leq n^{\prime} \leq n$ ) to buying $n^{\prime}-1$ number of journals if and only if the following inequality holds:

$$
n^{\prime} u+v\left(M-n^{\prime} p\right) \geq\left(n^{\prime}-1\right) u+v\left(M-n^{\prime} p+p\right)
$$


which is equivalent to

$$
u \geq U_{M B}\left(p, n^{\prime} p\right) .
$$

Lemma 1(i) implies that $U_{M B}\left(p, n^{\prime} p\right)$ strictly increases with $n^{\prime}$ and therefore it is optimal for the library to buy all the journals if and only if it prefers buying $n$ number of journals to buying $n-1$, a condition equivalent to $u \geq U_{M B}(p, n p)$.

We now prove that if $u<U_{M B}\left(\frac{M}{n}, M\right)=v\left(\frac{M}{n}\right)$, the unique $p^{*}$ satisfying $u=$ $U_{M B}\left(p^{*}, n p^{*}\right)$ is an equilibrium. Suppose that all publishers except $j$ charge $p^{*}$. If $j$ charges $p^{*}$, then all journals are sold and his profit is $p^{*}$; hence he has no incentive to choose a price lower than $p^{*}$. If instead he chooses $p_{j}\left(>p^{*}\right)$, then his journal is the most expensive one and the library will not buy it since $u<U_{M B}\left(p_{j},(n-1) p^{*}+p_{j}\right)$ holds by lemma 1(i).

In case $u \geq U_{M B}\left(\frac{M}{n}, M\right)$ holds, $p^{*}=\frac{M}{n}$ is an equilibrium. Still, publisher $j$ has no incentive to choose a price lower than $p^{*}$ since he can realize profit $p^{*}$ by charging $p^{*}$. If he chooses $p_{j}>p^{*}$, the library cannot afford to buy all the journals and, again, will drop $j$ 's journal because it is the most expensive.

The next proposition states that regardless of the level of industry concentration (with the exception of monopoly for some parameters), there exists a unique equilibrium and it is such that all publishers are active and all journals are sold at the same price $p^{*}$ determined above.

Proposition 1 (Irrelevance Result) Suppose that journals are homogeneous (i.e. $u_{i j}=$ $u>0$ for all $i j$ ) and are priced independently.

(i) For any level of industry concentration, there exists an equilibrium in which all publishers are active and all the journals are sold at the same price $p^{*}$, which is determined as follows: if $M \leq n v^{-1}(u), p^{*}=M / n$, and if $M>n v^{-1}(u), p^{*}$ is such that $n p^{*}<M$ and

$$
u=U_{M B}\left(p^{*}, n p^{*}\right) .
$$

(ii) The equilibrium is unique unless the industry is a monopoly and $M<n v^{-1}(u)$.

Proof. Here we prove (i); the proof of (ii) is given in the Appendix. Suppose that each publisher except $j$ charges price $p^{*}$ (as determined by the statement of the proposition) for any of his journals. We show that choosing $p^{*}$ for each journal is a best response for publisher $j$ having $n_{j}$ number of journals: the monopoly case is a special case with $n_{j}=n$. We first note that for any $\mathbf{p}_{j} \equiv\left(p_{1 j}, \ldots, p_{n_{j}}\right)$, the library will purchase any journal with price lower than or equal to $p^{*}$ because it is willing to buy $n$ number of journals at price $p^{*}$ : 
this follows from (2) if $M>v^{-1}(u)$ and from $u \geq U_{M B}\left(p^{*}, M\right)=v\left(\frac{M}{n}\right)$ if $M \leq n v^{-1}(u)$. Hence, for any $\mathbf{p}_{j}$ all the journals of any publisher $j^{\prime}(\neq j)$ will be purchased and $j$ 's profit is equal to $n_{j} p^{*}$ if he chooses $\mathbf{p}_{j} \equiv\left(p^{*}, \ldots, p^{*}\right)$. Therefore, our proof is done if we show that $j$ cannot achieve a profit higher than $n_{j} p^{*}$. This fact is obvious if $M \leq n v^{-1}(u)$, since for any $\mathbf{p}_{j}$ the library buys all the journals of the other publishers and therefore spends at most $M-\left(n-n_{j}\right) p^{*}=n_{j} p^{*}$ for the journals of $j$. For the case of $M>n v^{-1}(u)$, suppose that $j$ can realize a profit $\pi_{j}>n_{j} p^{*}$. Then it is necessary that the library buys a journal of $j$ with price $p^{\prime}>p^{*}$ and this requires $u \geq U_{M B}\left(p^{\prime},\left(n-n_{j}\right) p^{*}+\pi_{j}\right)$, but (2) and lemma 1(i) imply $u=U_{M B}\left(p^{*}, n p^{*}\right)<U_{M B}\left(p^{\prime},\left(n-n_{j}\right) p^{*}+\pi_{j}\right)$, a contradiction.

Proposition 1 establishes several results. First, each publisher is active independently of the number of journals he owns; since each journal has the same value, any publisher can make a positive profit by pricing his journals low enough to undercut the rivals' prices. Furthermore, all the journals have the same price $p^{*}$ such that the library buys all of them. In the case of a monopolist, charging the same price for all journals minimizes the competition from books. ${ }^{16}$ In the case of oligopoly (i.e. $N \geq 2$ ), if $p^{(1)}$ is the price of the most expensive journal that the library buys and $p_{i j}<p^{(1)}$, then publisher $j$ can increase his profit by suitably increasing the price of journal $i j$ and reducing the prices of all his other journals in a way which induces the library to buy the same journals of $j$ it purchased before, but at a higher total price. A similar argument can be used to show that publisher $j$ can increase his profit when some of his journals are not sold; therefore, all journals are sold in equilibrium.

Second, in equilibrium the competitive margin binds in that each publisher cannot raise the price of any of his journals without inducing the library to exclude the journal from the list of the journals it buys. We can further distinguish two cases depending on the way the margin binds. When the journal industry profit is smaller than $M$, the equilibrium price $p^{*}$ is determined by what we call marginal opportunity cost pricing in the following sense: the price $p^{*}$ is such that after purchasing $n-1$ number of journals at price $p^{*}$, the library is indifferent between buying an extra journal at price $p^{*}$ and spending $p^{*}$ instead on buying books. This is shown in Figure 2 in which the area of the rectangular $\mathrm{ABCD}$ is equal to $u .{ }^{17}$ When the journal industry profit is equal to $M$, the

\footnotetext{
${ }^{16}$ More precisely, given a profit $\pi$, for any price vector $\mathbf{p}$ with $\sum_{i=1}^{n} p_{i 1}=\pi$ and $\mathbf{p} \neq\left(\frac{\pi}{n}, \ldots, \frac{\pi}{n}\right)$, $\max _{i} U_{M B}\left(p_{i 1}, \pi\right)$ is strictly larger than $U_{M B}\left(\frac{\pi}{n}, \pi\right)$.

${ }^{17}$ The fact that each publisher regards his journal as the marginal one when choosing its price is similar to what happens in the literature on multilateral bargaining (Stole and Zweibel, 1996a,b, and Chemla, 2003). For instance, Chemla studies competition among downstream firms buying from an upstream one and finds that each downstream firm pays the price that the marginal firm would pay to the upstream
} 
equilibrium price $p^{*}(=M / n)$ satisfies what we call generalized marginal opportunity cost pricing in that each journal leaves to the library the same extra positive surplus with respect to its opportunity cost, which is equal to $u-v(M / n) \geq 0$. Generalized marginal opportunity cost pricing includes marginal opportunity cost pricing as a special case in which the extra surplus is equal to zero. In both cases, if a publisher increases the price of a journal from $p^{*}$, the library does not purchase it any more.

Finally, the irrelevance result states that the equilibrium price $p^{*}$ is the same for any level of industry concentration, except for the monopoly if $M<n v^{-1}(u)$. The result mainly comes from the two following facts. First, as was previously explained, regardless of the level of concentration, some publisher can increase his profit unless all journals have the same price. Second, the symmetric equilibrium price is uniquely determined by the condition that makes the competitive margin bind and this condition does not depend on industry concentration. Note that the uniqueness result does not hold in the case of monopoly with $M<n v^{-1}(u)$. Then, the monopolist can achieve profit $M$ not only by charging the uniform price $p^{*}=\frac{M}{n}$, but also with any price vector such that the sum of the prices is $M$ and the highest price $p^{\prime}$ satisfies $u \geq U_{M B}\left(p^{\prime}, M\right)$. Finally, we note that the equilibrium price $p^{*}$ depends on the number of journals in the industry and their value.

Example 1 Suppose $v(m)=31 m-m^{2}, M=10, u=42, n=3$; then $U_{M B}(p, \pi)=$ $p(31-p-2(M-\pi))$. By proposition 1, the equilibrium price $p^{*}$ under independent pricing is such that $U_{M B}\left(p^{*}, 3 p^{*}\right)=42$ since $v\left(\frac{M}{n}\right)>u$; thus $p^{*}=2$.

Remark 1 (robustness of the irrelevance result) Suppose that the library's utility from buying $k$ number of journals is $u(k)$ with $u(\cdot)$ increasing and concave. Then, we can show that the irrelevance result holds as long as $u(\cdot)$ is not very concave.

From the irrelevance result, we have the following corollary:

Corollary 1 When journals are homogeneous, under independent pricing

(i) no merger has any impact on (merging or non-merging) firms' profits and therefore firms have no strict incentive to merge;

(ii) no merger affects social welfare unless it creates a monopoly and $(n-1) v^{-1}(u) \geq M$.

Corollary 1(ii) deserves some explanation. If $k v^{-1}(u) \geq M$ for some $k \leq n-1$, then the monopolist can achieve profit $M$ by selling just $k$ number of journals instead of $n$.

one. However, none of these papers studies the issue of bundling. 


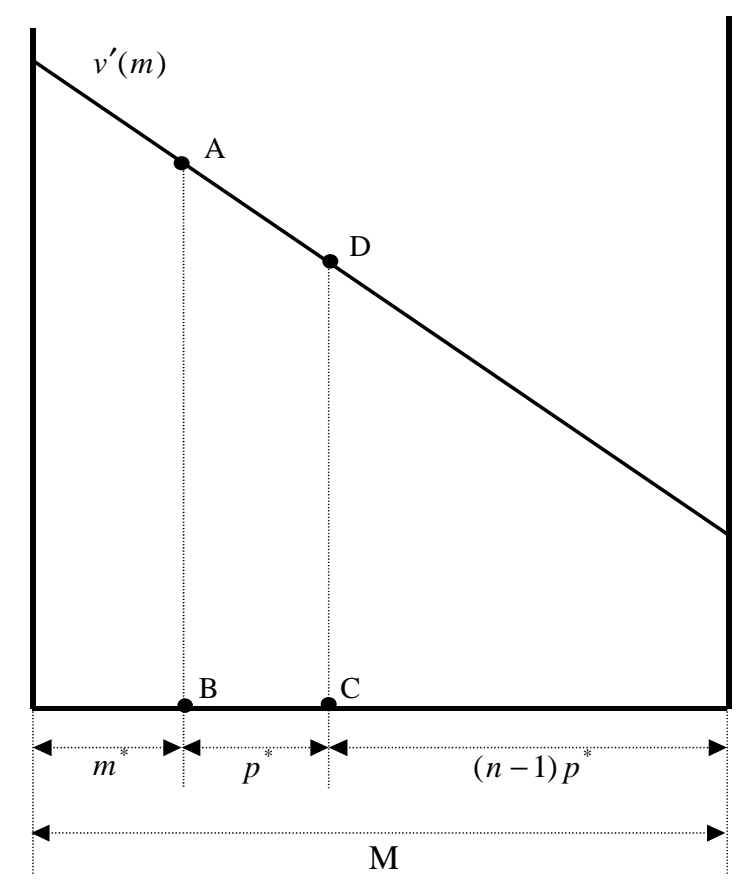

Figure 2: Equilibrium under independent pricing when $M>n v^{-1}(u)$ holds

Therefore, we cannot rule out the possibility that a merger which creates a monopolist reduces social welfare because less than $n$ journals are sold if $M$ is relatively small.

\subsection{Bundling}

In this subsection we analyze $\Gamma^{B}$, the game in which each active publisher bundles his journals and chooses a price for the bundle. Without loss of generality, we suppose that $U_{1} \geq U_{2} \geq \ldots \geq U_{N}$. Let $A^{*}$ represent the equilibrium set of active publishers, $\mathbf{P}^{*} \equiv\left\{P_{j}^{*}\right.$ : $\left.j \in A^{*}\right\}$ the equilibrium prices charged by the active publishers and $\pi^{B *} \equiv \sum_{j \in A^{*}} P_{j}^{*}$ the equilibrium industry profit in $\Gamma^{B}$.

Notice that our analysis does not depend on whether journals are homogeneous or heterogeneous because only the values $U_{1}, \ldots, U_{N}$ of the different bundles matter; therefore, the results in this subsection apply to the setting of heterogeneous journals as well. The next theorem characterizes the unique equilibrium of $\Gamma^{B}$.

Theorem 1 Under bundling, there exists a unique equilibrium and it is characterized as follows:

(i) If $M \leq v^{-1}\left(U_{1}-U_{2}\right)$, only the largest publisher is active and realizes profit $P_{1}^{*}=M$. 
(ii) If $M$ is such that there exists $k \in\{2, \ldots, N\}$ satisfying $\sum_{j=1}^{k-1} v^{-1}\left(U_{j}-U_{k}\right)<M \leq$ $\sum_{j=1}^{k} v^{-1}\left(U_{j}-U_{k+1}\right)$ (with $\left.U_{N+1} \equiv 0\right)$, only the $k$ largest publishers are active and charge prices which satisfy $\pi^{B *}=\sum_{j=1}^{k} P_{j}^{*}=M$ and

$$
U_{j}-U_{M B}\left(P_{j}^{*}, M\right)=U_{j^{\prime}}-U_{M B}\left(P_{j^{\prime}}^{*}, M\right) \geq U_{k+1} \quad \text { for any }\left\{j, j^{\prime}\right\} \subseteq A^{*} .
$$

(iii) If $M>\sum_{j=1}^{N} v^{-1}\left(U_{j}\right)$, all the publishers are active and charge prices which satisfy $\pi^{B *}=\sum_{j=1}^{N} P_{j}^{*}<M$ and

$$
U_{j}-U_{M B}\left(P_{j}^{*}, \pi^{B *}\right)=0 \quad j=1, \ldots, N
$$

We first note that the case of proposition 1 in which $n_{j}=1$ for any $j$ (i.e. each publisher owns only one journal) is a special case of parts (ii)-(iii) of this theorem with $U_{j}=u$ for all $j$ and $N=n$. Note also that all the bundles are sold if and only if the library's budget is large enough (i.e. $M>\sum_{j=1}^{N-1} v^{-1}\left(U_{j}-U_{N}\right)$ ). Otherwise, bundling makes small publishers unable to sell their journals while, under no-bundling, all journals are sold for any value of $M$. In what follows, we provide the main intuition about the equilibrium in $\Gamma^{B}$ by examining a special case with two publishers such that $U_{1}>U_{2}$.

Consider first the case in which the journal industry profit $\pi^{B *}$ is smaller than $M$; this implies that both publishers are active since otherwise an inactive publisher can make a profit by choosing a small price. Then, the equilibrium prices $\mathbf{P}^{*}=\left(P_{1}^{*}, P_{2}^{*}\right)$ are determined by marginal opportunity cost pricing as in $\Gamma^{I}$ when $M$ is large:

$$
U_{j}=U_{M B}\left(P_{j}^{*}, P_{1}^{*}+P_{2}^{*}\right), \text { for } j=1,2
$$

Publisher $j$ considers his bundle the marginal one and chooses $P_{j}^{*}$ such that the library is indifferent between buying $\mathrm{B} j$ and spending $P_{j}^{*}$ on buying extra books. In the special case in which $P_{1}^{*}+P_{2}^{*}$ is equal to $M$, we have $U_{M B}\left(P_{j}^{*}, P_{1}^{*}+P_{2}^{*}\right)=v\left(P_{j}^{*}\right)$; hence, from $(5), P_{j}^{*}=$ $v^{-1}\left(U_{j}\right)$. This suggests that a solution to (5) exists if and only if $M>v^{-1}\left(U_{1}\right)+v^{-1}\left(U_{2}\right)$, as Theorem 1(iii) states.

Second, consider the case in which the journal industry profit $\pi^{B *}$ is equal to $M$ and both bundles are sold. Then, $\mathbf{P}^{*}$ is determined by generalized marginal opportunity cost pricing as in $\Gamma^{I}$

$$
U_{1}-U_{M B}\left(P_{1}^{*}, M\right)=U_{2}-U_{M B}\left(P_{2}^{*}, M\right) \geq 0 \text {. }
$$

In other words, there is a kind of Bertrand competition such that the extra surplus is the same for all the bundles. The vector $\mathbf{P}^{*}$ constitutes an equilibrium since lowering $P_{j}$ is obviously suboptimal for publisher $j$ and if $P_{j}$ higher than $P_{j}^{*}$ is chosen, the library cannot 
afford to buy both bundles; it prefers dropping $\mathrm{B} j$ since at $\mathbf{P}^{*}$ it is indifferent between dropping B1 and dropping B2.

Finally, for a budget small enough we see that publisher 1's profit is equal to $M$, implying that publisher 2 cannot sell his bundle. This happens if $U_{1}-v(M) \geq U_{2}$ holds, because then, for any $P_{2}>0$, the library prefers buying B1 rather than B2 even if $P_{1}=M$ since the payoff $U_{1}$ from buying $\mathrm{B} 1$ is larger than $U_{2}+v\left(M-P_{2}\right)$, the payoff from buying B2 at $P_{2}$ and spending $M-P_{2}>0$ on books. The inequality $U_{1} \geq U_{2}+v(M)$ is equivalent to $M \leq v^{-1}\left(U_{1}-U_{2}\right)$, the condition in Theorem 1(i).

Example 2 Consider the parameters of example 1: $v(m)=31 m-m^{2}, M=10, u=42$ and $n=3$. Then, under bundling

(i) when $N=2$ and $n_{1}=2, n_{2}=1, P_{1}^{*}$ and $P_{2}^{*}$ satisfy

$$
\begin{aligned}
& U_{M B}\left(P_{1}^{*}, P_{1}^{*}+P_{2}^{*}\right)=84 \\
& U_{M B}\left(P_{2}^{*}, P_{1}^{*}+P_{2}^{*}\right)=42
\end{aligned}
$$

since $M>v^{-1}(2 u)+v^{-1}(u)$; hence $P_{1}^{*}=4.3655$ and $P_{2}^{*}=1.9382$. Notice that $P_{1}^{*}>2 p^{*}$, $p^{*}>P_{2}^{*}$ and $P_{1}^{*}+P_{2}^{*}>3 p^{*}$.

(ii) When $N=1$ and $n_{1}=3$, the monopolist chooses $P^{m}$ satisfying $U_{M B}\left(P^{m}, P^{m}\right)=126$ since $M>v^{-1}(3 u)$, hence $P^{m}=7$. Notice that $P^{m}>P_{1}^{*}+P_{2}^{*}>3 p^{*}$.

Theorem 1 and the discussion following the theorem show that marginal or generalized marginal opportunity cost pricing determines the prices under bundling in the same way as under independent pricing and therefore the competitive margin binds.

The competition between each bundle of journals and the marginal bundle of books implies that a large publisher (i.e. a publisher with high $U_{j}$ ) has a competitive advantage over a small publisher. Given $\pi^{B *}$, since $v^{\prime}(\cdot)$ is strictly decreasing, as the number of books in the marginal bundle increases, the average surplus of the books in this bundle decreases. Therefore, the marginal bundle of books competing with the bundle of a large publisher has a lower average surplus than the marginal bundle of books competing with the bundle of a small publisher. This fact implies a sort of economies of scale under bundling. The next corollary formalizes this intuition in two different, although related, ways. The first result shows that publisher $j$ 's profit per value of bundle $\frac{P_{j}^{*}}{U_{j}}$ strictly increases with the value $U_{j}$ of his bundle; the second result establishes that a large publisher gets a relatively large share of the industry profit. ${ }^{18}$

\footnotetext{
${ }^{18}$ The proof of the corollary is straightforward and hence is omitted.
} 
Corollary 2 Under bundling we have

(i) $\{j, h\} \subseteq A^{*}$ and $U_{j}>U_{h}$ imply $\frac{P_{j}^{*}}{U_{j}}>\frac{P_{h}^{*}}{U_{h}}$.

(ii) If $A \subset A^{*}$ is such that $U_{1} \geq \sum_{h \in A} U_{h}, 1 \notin A$ and $U_{1}>U_{2}$, then $P_{1}^{*}>\sum_{h \in A} P_{h}^{*}$.

We now use an example to discuss the role of our assumption about active and inactive publishers introduced in section 2.4.

Example 3 Consider the setting with $N=3, U_{1}=10, U_{2}=2, U_{3}=1.5, M=5, u(m)=$ $m+\sqrt{m}$. From the proof of Theorem 1 we know that there exists an equilibrium in $\Gamma^{B}$ in which publisher 3 makes a profit if and only if $M>v^{-1}\left(U_{1}-U_{3}\right)+v^{-1}\left(U_{2}-U_{3}\right){ }^{19}$ Since this inequality is violated with our parameters, there exists no equilibrium in which publisher 3 makes a positive profit. Without our assumption in section 2.4 that makes publisher 3 inactive (i.e. stay out of the market), we find infinitely many equilibria in $\Gamma^{B}$. For instance, $\tilde{\mathbf{P}}=(5-x, x, x)$ is an equilibrium for any $x \in(0,0.1]$ and in all these equilibria the library buys B1 and B2. ${ }^{20}$ There also exists an equilibrium $\hat{\mathbf{P}}=\left(P_{1}, P_{2}, P_{3}\right)=(5,5,5)$ in which the library buys only B1. Our assumption about active publishers eliminates all the infinite equilibria in which B1 and B2 are purchased and does not allow that the price chosen by a publisher which makes zero profits affects the equilibrium outcome. The consequence, for this particular example, is that only publisher 1 is active, as is predicted by theorem 1 since $M<v^{-1}\left(U_{1}-U_{2}\right)$.

\subsection{Incentive to bundle}

In the previous sections, we examined the two different regimes of no-bundling and bundling (games $\Gamma^{I}$ and $\Gamma^{B}$, respectively). In this section, we inquire which of these regimes will emerge endogenously in game $\Gamma$ by examining each publisher's incentive to bundle. We obtain the following result.

Proposition 2 (i) If publisher $j$ realizes profit $\pi_{j}>0$ by pricing journals independently, then he can earn the same profit by bundling his journals at price $P_{j}=\pi_{j}$.

(ii) In any equilibrium of $\Gamma$, any active publisher bundles his journals.

\footnotetext{
${ }^{19}$ See lemma 3(i) in appendix.

${ }^{20}$ The fact that $\tilde{\mathbf{P}}$ is an equilibrium can be verified by noticing that (i) if publisher 1 increases $P_{1}$ above $5-x$, then the library obtains a higher payoff by purchasing B2 and B3 $(3.5+5-2 x+\sqrt{5-2 x})$ rather than B1 $\left(10+5-P_{1}+\sqrt{5-P_{1}}\right)$; (ii) if publisher 2 increases $P_{2}$ above $x$, then the library buys B1 and B3 (payoff 11.5) rather than B2 and B3 $\left(3.5+5-x-P_{2}+\sqrt{5-x-P_{2}}\right)$ or only $\mathrm{B} 2\left(2+5-P_{2}+\sqrt{5-P_{2}}\right)$; (iii) if publisher 3 reduces $P_{3}$ to almost 0 , then the library buys B1 and B2 (payoff 12) rather than B1 and B3 $\left(11.5+x-P_{3}+\sqrt{x-P_{3}}\right)$ or B2 and B3 $\left(3.5+5-x-P_{3}+\sqrt{5-x-P_{3}}\right)$.
} 
This result says that any profit publisher $j$ can make without bundling his journals can also be obtained by bundling the journals; therefore, bundling is a weakly dominant strategy in $\Gamma$ for each publisher. However, this fact might be consistent with the existence of an equilibrium in $\Gamma$ in which one (or more) publisher(s) does not bundle. The second part of the proposition establishes that this is not the case.

Publishers have an incentive to bundle their journals since bundling has the direct effect of softening competition from books. In order to provide intuition, we consider a simple case of a monopolist owning two journals. Suppose that his profit under independent pricing is smaller than $M$. In this case, by Proposition 1 , in $\Gamma^{I}$ the monopolist chooses the same price $p^{*}$ for both journals, which is determined by marginal opportunity cost pricing:

$$
u-U_{M B}\left(p^{*}, 2 p^{*}\right)=0 .
$$

Suppose now that the monopolist bundles his journals. Consider first the case in which he charges price $2 p^{*}$ for the bundle. Then, (7) and lemma 1(ii) imply

$$
2 u-U_{M B}\left(2 p^{*}, 2 p^{*}\right)=2 U_{M B}\left(p^{*}, 2 p^{*}\right)-U_{M B}\left(2 p^{*}, 2 p^{*}\right)>0 .
$$

Under independent pricing, both journals compete with the same marginal bundle of books giving utility $U_{M B}\left(p^{*}, 2 p^{*}\right)$ to the library. In contrast, under bundling it is as if the first journal competes with the marginal bundle of books giving utility $U_{M B}\left(p^{*}, 2 p^{*}\right)$, while the second journal competes with the marginal bundle of books giving utility $U_{M B}\left(2 p^{*}, 2 p^{*}\right)-U_{M B}\left(p^{*}, 2 p^{*}\right)=U_{M B}\left(p^{*}, p^{*}\right)<U_{M B}\left(p^{*}, 2 p^{*}\right)$. This explains why the inequality in (8) holds and therefore there exists an $\varepsilon>0$ which satisfies

$$
2 u-U_{M B}\left(2 p^{*}+\varepsilon, 2 p^{*}+\varepsilon\right)>0 .
$$

This inequality shows that the library will buy the bundle if the monopolist charges $P=2 p^{*}+\varepsilon$ as the price for the bundle. Thus, bundling allows the monopolist to increase his profit with respect to independent pricing and the same intuition applies to the case where there is an oligopoly.

Remark $2{ }^{21}$ We derived the direct effect of bundling in a setting in which the utility from money is concave while the marginal utility from the consumption good (i.e. journals) is constant. The mechanism behind this direct effect is isomorphic to the mechanism behind two-part tariffs in a standard setting in which the marginal utility from money is constant

\footnotetext{
${ }^{21}$ We thank the referee for providing us with this idea.
} 
and the utility from the good is concave. For instance, consider a consumer with payoff $u(q)-t$, where $u(\cdot)$ is strictly increasing and strictly concave, $q$ is the quantity of the good he consumes and $t$ is his monetary payment. Suppose that a monopolist produces the good at a constant marginal cost $c(>0)$. In this case, independent pricing is equivalent to linear pricing and thus leaves some surplus to the consumer, but the monopolist can extract the full surplus with a suitable two-part tariff in which the marginal price is equal to $c$.

\subsection{Comparative Statics}

\subsubsection{Industry profit and social welfare}

Now we study the effect of bundling on industry profit and social welfare. Let $\pi^{I *}$ denote industry profits under independent pricing. We have:

Proposition 3 (i) If $M>n v^{-1}(u)$, bundling strictly increases industry profits: $\pi^{B *}>$ $\pi^{I *}$. If $M \leq n v^{-1}(u)$, bundling does not affect industry profits: $\pi^{B *}=\pi^{I *}=M$.

(ii) If $M>n v^{-1}(u)$, bundling strictly reduces social welfare by strictly reducing book consumption and weakly reducing journal consumption. If $M \leq n v^{-1}(u)$, bundling weakly reduces social welfare by weakly reducing journal consumption.

Proof. (i) From proposition 1 we know that $\pi^{I *}=M$ if $M \leq n v^{-1}(u)$. In contrast, theorem 1 shows that $\pi^{B *}=M$ when $M \leq \sum_{j=1}^{N} v^{-1}\left(U_{j}\right)$. Since $v^{-1}(0)=0$ and $v^{-1}$ is strictly convex, $n_{j} u=U_{j}$ implies $n_{j} v^{-1}(u)<v^{-1}\left(U_{j}\right)$ and in turn $n v^{-1}(u)<\sum_{j=1}^{N} v^{-1}\left(U_{j}\right)$; this proves the second part of the proposition (i).

Assume now $M>n v^{-1}(u)$, so that $\pi^{I *}<M$. If $M \leq \sum_{j=1}^{N} v^{-1}\left(U_{j}\right)$ holds, then $\pi^{B *}=M$ from theorem 1 and the proposition (i) trivially holds. Suppose in contrast that $M>$ $\sum_{j=1}^{N} v^{-1}\left(U_{j}\right)$, so that $\pi^{B *}<M$ by theorem 1 . In order to prove that $\pi^{B *}>\pi^{I *}$, we notice that for each publisher $j$ we have

$$
n_{j} U_{M B}\left(p^{*}, \pi^{I *}\right)=n_{j} u=U_{j}=U_{M B}\left(P_{j}^{*}, \pi^{B *}\right) .
$$

Define $P_{j}(\pi)$ by $U_{j} \equiv U_{M B}\left(P_{j}(\pi), \pi\right)$ and observe that $P_{j}($.$) is strictly decreasing by lemma$ 1(i). Furthermore, lemma 1(ii) and the first two equalities in (9) imply $P_{j}\left(\pi^{I *}\right)>n_{j} p^{*}$ for any $n_{j} \geq 2$. We now prove $\pi^{B *}>\pi^{I *}$ by contradiction. Suppose $\pi^{B *} \leq \pi^{I *}$. Since $P_{j}($.$) is strictly decreasing, we must have P_{j}^{*}=P_{j}\left(\pi^{B *}\right) \geq P_{j}\left(\pi^{I *}\right)$; this implies $\pi^{B *} \equiv$ $\sum_{j=1}^{N} P_{j}\left(\pi^{B *}\right) \geq \sum_{j=1}^{N} P_{j}\left(\pi^{I *}\right)>\sum_{j=1}^{N} n_{j} p^{*}=\pi^{I *}$, which is a contradiction. 
(ii) If $M>n v^{-1}(u)$, bundling strictly increases industry profits, implying that the library consumes less books in $\Gamma^{B}$ than in $\Gamma^{I}$. Furthermore, under no-bundling, the library buys all the journals while bundling can make small publishers unable to sell their journals as stated by theorem 1.

The result of proposition 3(i) is due to the direct effect of bundling in terms of softening competition from books. To provide intuition, we consider the case in which $\pi^{I *}<M$ and suppose that bundling does not increase industry profits: $\pi^{B *} \leq \pi^{I *}$. Then, the marginal bundle of books corresponding to any given price $p$ has a lower value under bundling than under independent pricing since $U_{M B}\left(p, \pi^{B *}\right) \leq U_{M B}\left(p, \pi^{I *}\right)$ holds from lemma 1(i). Therefore, from the direct effect, each publisher can make a higher profit under bundling than under independent pricing and hence we get a contradiction.

If publishers are symmetric in the sense that $U_{1}=\ldots=U_{N}$, then bundling increases the profit of each publisher. If instead publishers are asymmetric, the fact that bundling increases industry profits is bad news for small publishers who cannot benefit much from the direct effect of bundling since a publisher's bundling has an indirect effect of inflicting negative pecuniary externalities on the rival publishers. To provide intuition, we consider competition between a big publisher with $U_{1}=(n-N+1) u($ and $n>N)$ and $N-1$ number of small publishers with $U_{2}=\ldots=U_{N}=u$. We focus on the case in which $p^{*}<\frac{M}{n}$ in $\Gamma^{I}$ and compare $\Gamma^{I}$ with $\Gamma^{B}$. Obviously, no small publisher can benefit from bundling since he has only one journal. However, from proposition 3 , the big publisher's bundling increases industry profits: $\pi^{B *}>n p^{*}$. This inflicts negative externalities on all the small publishers since the marginal bundle of books corresponding to a given price of journal has a higher surplus after 1's bundling than before. For instance, if $\pi^{B *}<M$, each small publisher's profits in $\Gamma^{B}$ is $P_{2}^{*}$ with $U_{M B}\left(P_{2}^{*}, \pi^{B *}\right)=u=U_{M B}\left(p^{*}, n p^{*}\right)$; hence, from lemma $1(\mathrm{i}), P_{2}^{*}$ is smaller than $p^{*}$ since $\pi^{B *}>n p^{*}$. Furthermore, as we have seen in theorem 1 , these pecuniary externalities make the small publishers unable to sell their journals if $U_{1}$ is large enough to satisfy $M \leq v^{-1}\left(U_{1}-u\right)$. Since the fact that bundling increases industry profits implies that the library consumes fewer books in $\Gamma^{B}$ than in $\Gamma^{I}$, it follows that bundling reduces social welfare by reducing book and journal consumption.

Remark 3 (independent budget for journals): When the budget $M$ can be used either for the purchase of journals or kept in cash, we have a setting which is described formally by $v(m)=m$. Since most of the effects of bundling are based on the strict concavity of $v$, one might expect that bundling has no effect in this environment. In fact, this is true as long as $U \leq M$ since in this case the equilibrium price of a journal or a bundle is simply equal to its value. However, when $U>M$, bundling does not affect industry profits which 
are equal to $M$ but it can reduce social welfare by making small publishers unable to sell their journals. As theorem 1 shows, when industry profits are equal to $M$, there is a kind of Bertrand competition among bundles which makes the extra surplus that the library gets from a bundle with respect to its opportunity cost the same for all the bundles sold, creating an advantage for large publishers.

\subsubsection{Mergers}

We have seen that there is no incentive to merge under independent pricing. Here we study how bundling affects this incentive by considering a merger of two publishers. Let $\pi^{A M *}$ $\left(\pi^{B M *}\right)$ denote industry profits after the merger (before the merger) under bundling and $\pi_{j}^{B M *}$ publisher $j$ 's profit before the merger. Let $A^{B M *}$ denote the set of active publishers before the merger and let $\underline{j} \equiv \max \left\{j: j \in A^{B M *}\right\}$ denote the active publisher with the lowest valued bundle.

Proposition 4 Under bundling, consider a merger of any two publishers $j$ and $k$ such that $\{j, k\} \subset A^{B M *}$ and $\pi_{j}^{B M *}+\pi_{k}^{B M *}<M$.

(i) The merger strictly increases the joint profit of the merging publishers and strictly decreases the profit of any other publisher in $A^{B M *}$;

(ii) The merger strictly increases industry profits if $\pi^{B M *}<M$, otherwise $\pi^{A M *}=\pi^{B M *}=$ $M$.

(iii) The merger weakly reduces both book consumption (strictly if $\pi^{B M *}<M$ ) and journal consumption. Hence, it always weakly reduces social welfare.

The proposition says that a merger between any two active firms is strictly profitable unless the two firms already monopolize the market. As we mentioned above, in $\Gamma^{B}$ each bundle of journals competes with the marginal bundle of books and the average surplus of this bundle decreases as the number of books increases. Therefore, a large bundle of journals faces relatively soft competition from books. In this way, a merger increases profits of the merged publishers and industry profits. However, the fact that the library spends more money on the journals of the merging publishers imposes negative pecuniary externalities on all the other publishers, who therefore suffer a reduction in profits because of the merger. Regarding social welfare, since industry profits weakly increase as a consequence of the merger it is obvious that book consumption decreases. Furthermore, the merger may drive out of the market some publishers which were active before the merger (this suggests that the merger between Reed-Elsevier and Harcourt is 
likely to be anti-competitive), but cannot induce previously inactive publishers to become active. Hence, any merger among active publishers weakly reduces social welfare.

Remark 4 The only role played by a merger in our setting is that it allows the merging publishers to create a larger bundle. Therefore, if two or more publishers can sign an agreement to create a bundle of all their journals, this will have the same impact as a merger. As long as bundling is allowed, small publishers have an incentive to form a big bundle by signing such an agreement. This can improve social welfare if big publishers' bundling would make the small publishers unable to sell their journals without the agreement. ${ }^{22}$

\subsection{Bundling and incentive to acquire a journal}

In this section, we study how bundling affects publishers' incentives to acquire a journal sold by a third party. Our previous results have shown that bundling can make small publishers unable to sell their journals. This may induce them to exit the market and to sell their journals to other publishers. Alternatively, the journal on sale can be interpreted as a new journal. Under this interpretation, we study how bundling affects the incentive to introduce a new journal by examining which publisher has the highest willingness to pay for it.

There are $n$ number of journals before a third-party sells a journal with value $u$ through a second-price auction; $b_{j}$ represents the bid of publisher $j$. When each bidder knows before the auction the value he attaches to the good on sale (the so-called setting of private values), it is well known that there exists a unique weakly dominant strategy for him: bidding his own valuation for the good. In our setting, however, a bidder's value for the auctioned journal is given by the difference between his profit if he wins and his profit if some other publishers wins the journal. Since the latter profit may depend on the identity of the winning publisher, a bidder may have no dominant strategy and this makes the analysis more complicated with respect to a standard second price auction. However, we know that under independent pricing the equilibrium prices do not depend on the industry structure; therefore, a dominant strategy equilibrium exists. Likewise, under bundling, if there are two publishers, it is common knowledge that if publisher 1 (2) does not win the journal, then publisher 2 (1) wins ${ }^{23}$ and a dominant strategy exists here

\footnotetext{
${ }^{22}$ The agreement does not reduce book consumption since the fact that some publishers were inactive before the agreement requires industry profits before the agreement to be equal to $M$.

${ }^{23}$ We do not consider reserve prices or other instruments which may leave journal in the hands of the
} 
as well. For simplicity, we assume that before the auction all publishers are active under bundling, $\pi^{B}<M$ and $U_{1}>U_{2}$. Obviously, industry profits (weakly) increase after the auction. Under bundling, let $\pi^{B j}$ denote industry profits in the case in which publisher $j$ wins the auction.

We obtain the following result:

Proposition 5 Suppose a third-party sells a journal of value $u$ through the second-price auction.

(i) In the unique undominated equilibrium under independent pricing, all publishers make the same bid.

(ii) When $N=2$, in the unique undominated equilibrium under bundling we have $b_{1}>b_{2}$ if $\pi^{B 2}<M$ and $b_{1}=b_{2}$ if $\pi^{B 2}=M$. When $N \geq 3$, under bundling, bidder 1 wins the auction in any undominated equilibrium.

Proof. We prove (i) here: see Appendix for the proof of (ii). Let $p^{*}(n)$ denote the equilibrium price in $\Gamma^{I}$ described in proposition 1 as a function of the number of journals. If publisher $j$ wins the auction, we know from Proposition 1 that he will sell to the library all of his $n_{j}+1$ journals at the uniform price $p^{*}(n+1)$, thus realizing profit $\left(n_{j}+1\right) p^{*}(n+1)$. If instead publisher $j$ loses the auction, another publisher will win the journal but the equilibrium price will still be $p^{*}(n+1)$; j's profit will be $n_{j} p^{*}(n+1)$. Therefore, the increase in publisher $j$ 's profit from winning the auction with respect to losing it is $p^{*}(n+1)$, for $j=1, \ldots, N$, regardless of the identity of the winner. This implies that publishers $j$ 's valuation is $p^{*}(n+1)$, for $j=1, \ldots, N$. Therefore each publisher has a (unique) weakly dominant strategy, which is $b_{j}=p^{*}(n+1)$ for $j=1, \ldots, N$.

This proposition implies that bundling could have a serious impact on the evolution of the industry concentration. In the absence of bundling, publishers have the same willingness to pay for the auctioned journal. In contrast, under bundling, the largest publisher has always the highest willingness to pay for the journal. Although a more careful analysis needs to be undertaken to make a prediction on the industry dynamics, our result suggests that bundling might create a vicious circle through which big publishers induce exit of small publishers and become even bigger by purchasing their titles.

auctioneer. 


\section{The general case of heterogeneous journals}

In this section we consider the general case in which journals can have different values. Since theorem 1 applies to $\Gamma^{B}$, we only need to study $\Gamma^{I}$. Concerning the analysis of $\Gamma^{I}$, we find that the irrelevance result holds when we consider the two extreme cases of maximum industry concentration (when there is a monopolist) and minimum industry concentration (when each publisher owns only one journal). For the intermediate setting of oligopoly, the problem which was mentioned at the end of subsection 3.2 arises: the prices of the journals that are bought by the library might be affected by the prices of the journals that are not bought. In particular, a publisher may choose the prices for his unsold journals in a way which maximizes his profit from the journals he is able to sell; this makes the analysis very complicated. For the sake of tractability, we eliminate this problem by assuming that each publisher chooses from the set of his journals a subset of active journals and makes a journal active only if he expects it to be sold at a strictly positive price and that the library can purchase only active journals. This assumption is stronger than the one introduced in section 2.4 since in the latter case we allow a publisher to post prices for all his journals as long as the library buys at least one of them.

Under this assumption on active journals, there exists a unique equilibrium candidate (in pure strategies) regardless of the level of industry concentration; therefore, if the equilibrium exists, the irrelevance result holds. The equilibrium exists under the minimum and the maximum industry concentration but, for intermediate levels of concentration, it may not exist; we provide a sufficient condition for existence and an example of nonexistence. $^{24}$

The equilibrium of $\Gamma^{I}$ when each publisher owns only one journal (i.e. $N=n$ ) can be obtained from theorem 1 by replacing $U_{j}$ with $u_{1 j}$, where $u_{1 j}$ represents the value of the unique journal owned by publisher $j$.

Corollary 3 Under independent pricing, in the n-publisher-n-journal setting, there exists a unique equilibrium and it is characterized as follows:

(i) If $M \leq v^{-1}\left(u_{11}-u_{12}\right)$, only the largest publisher is active and realizes profit $p_{11}^{*}=M$.

(ii) If $M$ is such that there exists $k \in\{2, \ldots, n\}$ satisfying $\sum_{j=1}^{k-1} v^{-1}\left(u_{1 j}-u_{1 k}\right)<M \leq$ $\sum_{j=1}^{k} v^{-1}\left(u_{1 j}-u_{1 k+1}\right)\left(\right.$ with $\left.u_{1 n+1} \equiv 0\right)$, only the $k$ largest publishers are active and charge prices such that $\sum_{j=1}^{k} p_{1 j}^{*}=M$ and

$$
u_{1 j}-U_{M B}\left(p_{1 j}^{*}, M\right)=u_{1 j^{\prime}}-U_{M B}\left(p_{1 j^{\prime}}^{*}, M\right) \geq u_{1 k+1} \quad \text { for any }\left\{j, j^{\prime}\right\} \subset\{1,2, \ldots, k\} .
$$

\footnotetext{
${ }^{24}$ Notice that non-existence in the example does not depend on the assumption about active journals.
} 
(iii) If $M>\sum_{j=1}^{n} v^{-1}\left(u_{1 j}\right)$, all publishers are active and charge prices which satisfy $\pi^{*}=\sum_{j=1}^{n} p_{1 j}^{*}<M$ and

$$
u_{1 j}-U_{M B}\left(p_{1 j}^{*}, \pi^{*}\right)=0 \quad j=1, \ldots, n
$$

Let $u^{(k)}$ be the value of the journal with the $k$-highest value; hence, $u^{(1)} \geq \ldots \geq u^{(n)}$. The next theorem covers the general case in which at least one publisher owns two or more journals.

Theorem 2 Under independent pricing

(i) If $N=1$, the monopolist's profit is smaller than $M$ if $M>\sum_{i=1}^{n} v^{-1}\left(u_{i 1}\right)$ and equal to $M$ otherwise. The equilibrium prices in the $n$-publisher-n-journal setting (corollary 3(ii)-(iii)) maximize the monopolist's profit for any value of $M$; they are the unique profit maximizing prices if and only if $M \geq \sum_{i=1}^{n} v^{-1}\left(u_{i 1}\right)$.

(ii) In the case of oligopoly, under the assumption about active journals,

(a) there exists a unique equilibrium candidate regardless of the level of industry concentration, which is the equilibrium in the n-publisher-n-journal setting;

(b) the equilibrium exists if journals are nearly homogeneous.

Theorem 2(i) says that the level of industry concentration does not affect the outcome in the two extreme cases of minimum and maximum concentration as long as the industry profit is lower than $M .{ }^{25}$ Furthermore, theorem 2(ii)(a) establishes that the outcome does not depend on the level of industry concentration when $1<N<n$ as long as $\Gamma^{I}$ has an equilibrium. In the proof of theorem 2(ii)(b), we show that the equilibrium exists if journals are nearly homogeneous (therefore, proposition 1 which deals with the case of homogeneous journals is a special case of theorem 2). The equilibrium may not exist since a multi-journal publisher may change several prices at the same time and this deviation from the unique candidate price vector is sometimes profitable, as in the next example.

Example 4 Suppose that $v(m)=m+4 \sqrt{m}, M=12, N=2, n_{1}=2, n_{2}=1, u_{11}=u_{21}=$ 1 and $u_{12}=24$. Since $v(11)>24$ and $v\left(\frac{1}{2}\right)>1$, we infer, respectively, that $11>v^{-1}(24)$ and $\frac{1}{2}>v^{-1}(1)$. Hence, $M=12>\sum_{h=1}^{3} v^{-1}\left(u^{(h)}\right)$ and in the candidate equilibrium all journals are active with prices such that $p_{11}+p_{21}+p_{12}<12,1=U_{M B}\left(p_{11}, \pi\right)=$ $U_{M B}\left(p_{21}, \pi\right)$ and $24=U_{M B}\left(p_{12}, \pi\right) ;$ this yields $p_{11}^{*}=p_{21}^{*} \simeq 0.17$ and $p_{12}^{*} \simeq 11.566$.

\footnotetext{
${ }^{25} \mathrm{As}$ in the case of homogeneous journals, when $\pi^{I *}=M$ for some parameters it is possible for the monopolist to realize a profit equal to $M$ by selling a strict subset of the journals that are purchased by the library in the $n$-publisher- $n$-journal setting.
} 
However, a profitable deviation for publisher 1 is to set $p_{11}=p_{11}^{*}+0.05$ and $p_{21}=p_{21}^{*}+0.05$ because then the library's payoff is maximized by purchasing only publisher 1 's journals. ${ }^{26}$

This example is somewhat counterintuitive, since the library buys the three journals under prices $\mathbf{p}^{*}$ but it buys journals 11 and 21 and not journal 12 after $p_{11}$ and $p_{21}$ increase while $p_{12}$ does not change. Note first that conditional on the fact that journals 11 and 21 are purchased at prices $p_{11}=p_{11}^{*}+\varepsilon_{1}>p_{11}^{*}$ and $p_{21}=p_{21}^{*}+\varepsilon_{2}>p_{21}^{*}$, pecuniary externalities imply that the library does not buy journal 12 because $u_{12}=U_{M B}\left(p_{12}^{*}, p_{11}^{*}+p_{21}^{*}+p_{12}^{*}\right)$ while $u_{12}<U_{M B}\left(p_{12}^{*}, p_{11}^{*}+\varepsilon_{1}+p_{21}^{*}+\varepsilon_{2}+p_{12}^{*}\right)$. However, given that it is suboptimal to buy all the three journals, it is puzzling that the dropped journal is the one whose price is unchanged. Comparing the payoffs from the different alternatives sheds light on this issue; consider $\varepsilon_{1}=\varepsilon_{2}=\varepsilon$. If the library buys journals 11 and 21, its payoff is reduced by $v\left(M-\pi+p_{12}^{*}\right)-v\left(M-\pi+p_{12}^{*}-2 \varepsilon\right)>0$ with respect to the payoff before the changes in prices; if journals 11 and 12 (or 21 and 12) are purchased, the library's payoff decreases by $v\left(M-\pi+p_{11}^{*}\right)-v\left(M-\pi+p_{11}^{*}-\varepsilon\right)>0$. Therefore, journal 12 is eliminated if $v\left(M-\pi+p_{12}^{*}\right)-v\left(M-\pi+p_{12}^{*}-2 \varepsilon\right)<v\left(M-\pi+p_{11}^{*}\right)-v\left(M-\pi+p_{11}^{*}-\varepsilon\right)$. Even though $2 \varepsilon>\varepsilon>0$, if $p_{12}^{*}>p_{11}^{*}$ it is possible that the inequality holds for some $\varepsilon$ because of the strict concavity of $v$; in particular, it holds for $\varepsilon=0.05$. In words, there is much more money left for books when an expensive journal like 12 is dropped than when a cheap one like 11 or 21 is dropped. Therefore, the utility loss from spending $2 \varepsilon$ less money on books in the former case can be smaller than the utility loss from spending $\varepsilon$ less money in the latter case.

As we mentioned above, theorem 1 on $\Gamma^{B}$ is valid regardless of whether journals are homogeneous or heterogeneous. Furthermore, in $\Gamma$, i.e. the game in which each publisher chooses between bundling and no-bundling, there always exists an equilibrium in which every active publisher bundles his journals and $A^{*}$ and $\mathbf{P}^{*}$ are determined by theorem $1^{27}$. Therefore, conditional on equilibrium existence in $\Gamma^{I}$, most of the results that we obtained in the case of homogeneous journals hold in the general case of heterogeneous journals as well: the results regarding the incentive to bundle, the effect of bundling on profits and mergers and the incentive to acquire a journal. Concerning how bundling affects

\footnotetext{
${ }^{26}$ First, notice that after the deviation of publisher 1 it is infeasible to buy all the journals since the sum of prices is larger than $M$. The payoff from buying journals 11 and 21 is $2+12-2 * 0.22+4 \sqrt{12-2 * 0.22}=$ 27. 16; the payoff from buying 11 and 12 (or 21 and 12) is $25+12-0.22-11.566+4 \sqrt{12-0.22-11.566}=$ 27.064 ; the payoff from buying only 12 is $24+12-11.566+4 \sqrt{12-11.566}=27.069$.

${ }^{27}$ Note also that any equilibrium of $\Gamma$ in which a publisher does not bundle his journals requires the use of a weakly dominated strategy since a result similar to proposition 2(i) holds.
} 
social welfare, we have to distinguish its impact on book consumption from the impact on journal consumption. Bundling decreases book consumption since the direct effect of softening competition from books increases industry profits. Whether bundling decreases or increases journal consumption depends on the degree of symmetry among publishers. For instance, in the extreme case of symmetric publishers with $U_{1}=\ldots=U_{N}$, bundling may increase journal consumption since all bundles will be sold whereas journals of small value might not be sold under independent pricing when journals are very heterogeneous. However, this scenario is not realistic given that a small number of commercial publishers own a large number of journals; ${ }^{28}$ for instance, Reed-Elsevier alone has about 1800 journals. Since asymmetry among bundles is likely to be larger than asymmetry among individual journals, bundling is expected to reduce journal consumption making it hard for small publishers to sell their journals even if their quality is high. ${ }^{29}$

\section{Concluding remarks}

Our analysis reveals that there is a strong conflict between private and social incentives in the bundling of e-journals; each publisher wants to bundle his journals and bundling increases industry profits but reduces social welfare. In particular, big publishers' bundling not only reduces consumption of monographs but also can make small publishers unable to sell their journals even though they own high-quality journals. In this respect, it is noteworthy that Wolters Kluwer, which is the sixth-largest player in the industry by revenues, recently opted to exit scientific publishing and to focus solely on medical publishing, citing lack of scale as the reason for the exit (Gooden et al. 2002).

We found that bundling has two other important effects. First, bundling creates incentives for mergers. However, mergers among active publishers reduce social welfare by reducing book and journal consumption. In contrast, mergers among publishers who would not be able to sell their journals because of their lack of size might increase social welfare. Alternatively, it would be desirable for small publishers who have high-quality journals to sell their journals through a common agency as in the case of JSTOR. Second, bundling can have a serious impact on the evolution of industry concentration by affecting

\footnotetext{
${ }^{28}$ Measured by revenue, in 2001 Elsevier Science had a 16.0 percent industry share, Kluwer, 8.2 percent and Thomson-Scientific \& Healthcare, 7.5 percent (Edlin and Rubinfeld, 2004).

${ }^{29}$ Actually, some publishers think that if they are below number five in the shopping list of libraries, there is no guarantee that there will be any money left in the budget of the libraries (Key Perspectives, 2002).
} 
the incentives to acquire other journals. We have shown that in the absence of bundling, each publisher has the same willingness to pay for a journal while, under bundling, the largest publisher has always the highest willingness to pay. Hence, bundling might create a vicious cycle through which big publishers induce the exit of small publishers and become even bigger by purchasing their titles.

We studied how bundling affects a library's purchase of journals and books when its budget is given. It would be interesting to study how bundling affects the choice of the budget. For instance, one can consider the case in which the university of a library is a first mover and can set the budget before publishers make any decision. Although a complete analysis of this question is beyond the scope of this paper, we found that in the case of heterogeneous journals (conditional on equilibrium existence under independent pricing), bundling has an ambiguous effect on the university's incentive to increase the library's budget with respect to independent pricing (and therefore, it is possible that bundling induces the university to increase the budget). ${ }^{30}$

Finally, it would be interesting to extend our framework to other economic situations such as bundling (or block booking) in distribution of movies, TV or radio programs ${ }^{31}$. A rationale for the per se illegal status of block booking ${ }^{32}$ comes from the concern that block booking of high-quality movies with low-quality ones would make it difficult for small producers to get their high-quality movies into theaters. Our analysis shows that the above concern is justified at least in the market for e-journals.

\section{References}

[1] Armstrong, M. (1996). "Multiproduct Nonlinear Pricing". Econometrica 64(1): 5175 .

[2] Armstrong, M. (1999). "Price Discrimination by a Many-Product Firm", Review of Economic Studies, 66: 151-168.

\footnotetext{
${ }^{30}$ Both under independent pricing and bundling, as the library's budget increases, its payoff is piecewise constant and jumps upwards at a number of points and then it increases in a continuous way. In the case of bundling, the jumps occur less frequently but are larger.

${ }^{31}$ Since we assume price discrimination based on usage, our explanation of bundling is very different from the one based on second-degree price discrimination given by Stigler (1968).

${ }^{32}$ For the antitrust cases, see United States v. Paramount Pictures, Inc. et al. (1948) and United States v. Loew's, Inc. Et al. (1962). In MCA Television Ltd. v. Public Interest Corp. (11th Circuit, April 1999), the court of appeals reaffirmed the per se illegal status of block booking.
} 
[3] Bakos, Yannis and Eric Brynjolfsson (1999). "Bundling Information Goods: Pricing, Profits and Efficiency" Management Science, December

[4] Bakos, Yannis and Eric Brynjolfsson (2000). "Bundling and Competition on the Internet: Aggregation Strategies for Information Goods" Marketing Science, January

[5] Bergstrom, Theodore C. (2001). "Free Labor for Costly Journals." Journal of Economic Perspectives, Fall 15(4): 183-98

[6] Bolman, Pieter S. H. (2002). "The Consortium Site Licence: Unfinished Business?" The Consortium Site licence: Is It a Sustainable Model? Ingenta Institute, 241-46

[7] Chemla, Gilles (2003). "Downstream Competition, Foreclosure and Vertical Integration" Journal of Economics and Management Strategy, 12(2): 261-89

[8] Edlin, Aaron S. and Daniel L. Rubinfeld (2004). "Exclusion or Efficient Pricing: The "Big Deal" Bundling of Academic Journals", Antitrust Law Journal, 72(1): 119-157.

[9] Frazier, Kenneth (2001). "The Librarians' Dilemma: Contemplating the Costs of the Big Deal". D-Lib Magazine 7 (March 3,). http://www.dlib.org/dlib/march01/frazier/03frazier.html

[10] Gooden Paul, Matthew Owen and Sarah Simon (2002). "Scientific Publishing: Knowledge is Power" Morgan Stanley Equity Research: Europe: Industry: Media, September 27

[11] Haank, Derk (2001). "Is electronic publishing being used in the best interests of science? The Publisher's view". Proceedings of the Second ICSU-UNESCO International Conference on Electronic Publishing in Science

[12] Key Perspectives (2002). "Library Consortia Research: Library and Publisher studies" The Consortium Site licence: Is It a Sustainable Model? Ingenta Institute, 80-118

[13] Kyrillidou, Martha. (1999). "Spending More for Less". ARL Bimonthly Report, June (204).

[14] Lerner, J. and J. Tirole. (2004). "Efficient Patent Pools". American Economic Review, 94(3): 691-711. 
[15] Mas-Colell, A., M.D. Whinston and J.R. Green (1995), Microeconomic Theory, New York, N.Y.: Oxford University Press.

[16] McAfee, R. P., J. McMillan, M. D. Whinston. (1989). "Multiproduct Monopoly, Commodity Bundling, and Correlation of Values". Quarterly Journal of Economics. 114(May): 371-384

[17] McCabe, Mark J. (2002a). "Journal Pricing and Mergers: A Portfolio Approach". American Economic Review, 92:259-269

[18] McCabe, Mark J. (2002b). "Portfolio Models of Journal Pricing: Print v. Digital" Working paper, Georgia Institute of Technology

[19] McCabe, Mark J. Christopher Snyder, (2004). "The Economics of Open Access Journals" Working paper, Georgia Institute of Technology and George Washington University

[20] Nalebuff, Barry. (2004). "Bundling as an Entry Barrier". Quarterly Journal of Economics, 119(1):159-188

[21] Salinger, M. A. (1995). "A Graphical Analysis of Bundling". Journal of Business. 68(1):85-98

[22] Schmalensee, R. L. (1984). "Gaussian Demand and Commodity Bundling". Journal of Business. 57: S211-S230

[23] Stigler, G. J. (1968). "A Note on Block Booking." in G.J. Stigler (ed.), The Organization of Industries. Homewood, Ill.: Irwin.

[24] Stole, Lars A. and Jeffrey Zweibel (1996a). "Intra-Firm Bargaining under Nonbinding Contracts." Review of Economic Studies, 63: 375-410

[25] Stole, Lars A. and Jeffrey Zweibel (1996b). "Organizational Design and Technology Choice under Intrafirm Bargaining." American Economic Review, 86: 195-222

[26] U.K. Competition Commission. (2001). Reed Elsevier Plc and Harcourt General, Inc: $A$ report on the proposed merger. U.K.

[27] Whinston, M. D. (1990). "Tying, Foreclosure and Exclusion". American Economic Review, 80: 837-59. 


\section{Appendix \\ Proof of Proposition 1(ii)}

We note first that in the case of the monopolist with $M \geq n v^{-1}(u)$, charging the uniform price $p^{*}$ is the only way to achieve the maximum profit $n p^{*}$ since, otherwise, we have a contradiction; the most expensive journal among the ones the library buys has price $p^{(1)}$ strictly higher than $p^{*}$ but actually it will not be purchased since $u=U_{M B}\left(p^{*}, n p^{*}\right)<$ $U_{M B}\left(p^{(1)}, n p^{*}\right)$ holds. Therefore, in what follows, we focus on the case of $N \geq 2$. The proof is composed of three claims. It is useful to recall that, given the prices chosen by active publishers, it is optimal for the library to buy the $n^{\prime}(\leq n)$ cheapest journals, where $n^{\prime}$ is endogenous.

Claim 1 All publishers are active in any equilibrium of $\Gamma^{I}$.

Proof Suppose that publisher $h$ is not active. Then, we can easily show that he can make a positive profit if he becomes active. Given the prices of the journals of active publishers, let publisher $h$ choose the same price $\varepsilon(>0)$ for each of his journals, small enough to make them cheaper than any journal of other active publishers. Since $u>U_{M B}\left(\varepsilon, n_{h} \varepsilon\right)$ for a small $\varepsilon$, the library buys all the journals of publisher $h$ and he makes a profit $n_{h} \varepsilon>0$. $\diamond$

Claim 2 If $N \geq 2$, in any equilibrium of $\Gamma^{I}$ every journal has the same price, denoted by $\widehat{p}$, and all the journals are purchased by the library.

Proof For any $j$, let $R_{j}\left(Z_{j}\right)$ be the set of his journals which are sold (not sold); $R \equiv$ $\cup_{j=1}^{N} R_{j}$ and $Z \equiv \cup_{j=1}^{N} Z_{j}$. We first prove that all journals in $R$ have the same price. Let $p^{(1)} \equiv \max _{i j \in R}\left\{p_{i j}\right\}$ denote the price of the most expensive journal the library buys; then, $u \geq U_{M B}\left(p^{(1)}, \pi\right)$. We prove that if $p_{i h}<p^{(1)}$ for some $i h \in R$, publisher $h$ can increase his profit by increasing the price of journal $i h$ by $\varepsilon(>0)$ small enough and reducing by $\frac{\varepsilon}{\left|R_{h}\right|}$ the price of each other journal in $R_{h}$, where $\left|R_{h}\right|$ is the number of journals in $R_{h}$ : let $\tilde{p}_{i h}=p_{i h}+\varepsilon$ and $\tilde{p}_{i^{\prime} h}=p_{i^{\prime} h}-\frac{\varepsilon}{\left|R_{h}\right|}$ for any $i^{\prime} h \in R_{h} \backslash\{i h\} .{ }^{33}$ At the new prices, it is obvious that the library buys all the journals in $R_{h}$ if at least one journal with price $p^{(1)}$ is still purchased, since each journal in $R_{h}$ has a price smaller than $p^{(1)}$. If instead no journal with price $p^{(1)}$ is purchased, then also no journal in $Z$ is purchased since $p_{i j} \geq p^{(1)}$ for any $i j \in Z$. Therefore, an upper bound for the new industry profit is $\pi-p^{(1)}+\varepsilon$, where $\pi$ is the industry profit before publisher $h$ 's prices change. This implies that all the journals in $R_{h}$ are purchased since $\tilde{p}_{h}^{(1)} \equiv \max _{i^{\prime} h \in R_{h}}\left\{\tilde{p}_{i^{\prime} h}\right\}<p^{(1)}, \pi-p^{(1)}+\varepsilon<\pi$ and $u \geq U_{M B}\left(p^{(1)}, \pi\right)$ imply $u>U_{M B}\left(\tilde{p}_{h}^{(1)}, \pi-p^{(1)}+\varepsilon\right)$.

\footnotetext{
${ }^{33}$ Notice that without loss of generality we can assume that some publisher $j \neq h$ owns a journal with price $p^{(1)}$ which is purchased by the library.
} 
Now we prove that all the journals are sold. Suppose that $i h \in Z$ for some $i$ and $h$. Then, publisher $h$ can increase his profit by setting $p_{i h}=\varepsilon(>0)$ and reducing the price of each journal in $R_{h}$ by $\frac{\varepsilon}{1+\left|R_{h}\right|}$. In this way, all the journals in $R_{h} \cup\{i h\}$ are purchased since they are cheaper then any journal in $\cup_{j \neq h} R_{j}$ and the logic of the proof in the previous paragraph applies.

Claim 3 If $N \geq 2$, in any equilibrium of $\Gamma^{I}, \widehat{p}$ is equal to $p^{*}$ described by the proposition.

Proof The library buys all the journals at price $\widehat{p}$ if and only if $u \geq U_{M B}(\widehat{p}, n \widehat{p})$ and $n \widehat{p} \leq M$. Consider first the case of $M>n v^{-1}(u)$. Then $u<U_{M B}(M / n, M)(=v(M / n))$ holds and therefore we must have $\widehat{p}<M / n$. We now show that $\widehat{p}<M / n$ implies $u=U_{M B}(\widehat{p}, n \widehat{p})$. To prove this, suppose that $\widehat{p}<M / n$ and $u>U_{M B}(\widehat{p}, n \widehat{p})$ hold. Then, let a publisher $h$ increase the price of one of his journals to $\widehat{p}+\varepsilon$ with $\varepsilon(>0)$ small enough. In this case, all journals of all publishers are still sold because $u>U_{M B}(\widehat{p}, n \hat{p})$ implies $u>U_{M B}(\widehat{p}+\varepsilon, n \widehat{p}+\varepsilon)$. Finally, $u=U_{M B}(\widehat{p}, n \widehat{p})$ has a unique solution smaller than $M / n$, given that $M>n v^{-1}(u)$.

In the case of $M \leq n v^{-1}(u), \widehat{p}$ must be equal to $M / n$ because $u>U_{M B}(p, n p)$ for any $p<M / n$.

\section{Proof of Lemma 2}

Lemma 2 Let $S$ denote a set of items ${ }^{34}$ with $P_{S}=\sum_{s \in S} p_{s} \leq M$. If only the items in $S$ are considered for purchase, then all of them are purchased if and only if

$$
u_{s} \geq U_{M B}\left(p_{s}, P_{S}\right) \quad \text { for all } \quad s \in S
$$

Proof. Let $U_{S} \equiv \sum_{s \in S} u_{s}$.

$(\Rightarrow)$ If buying all the items in $S$ is optimal, then buying all gives a higher utility than buying all except a particular item $s$, for any $s \in S$. Therefore, the following condition must be satisfied:

$$
U_{S}+v\left(M-P_{S}\right) \geq U_{S}-u_{s}+v\left(M-P_{S}+p_{s}\right), \quad \text { for all } s \in S
$$

which is equivalent to (10).

\footnotetext{
${ }^{34}$ An item can be either a journal or a bundle since Lemma 2 applies to the general case in which some publishers bundle their journals while the others don't.
} 
$(\Leftarrow)$ Let $S^{\prime} \subseteq S$ denote a subset of $S$ with $P_{S^{\prime}}=\sum_{s \in S^{\prime}} p_{s}$ and $U_{S^{\prime}} \equiv \sum_{s \in S^{\prime}} u_{s}$. Suppose that (10) holds, which implies

$$
U_{S^{\prime}}=\sum_{s \in S^{\prime}} u_{s} \geq \sum_{s \in S^{\prime}} U_{M B}\left(p_{s}, P_{S}\right) \geq U_{M B}\left(P_{S^{\prime}}, P_{S}\right)
$$

where the second inequality holds because $U_{M B}\left(p_{s}, P_{S}\right)$ is concave in the first argument and $U_{M B}\left(0, P_{S}\right)=0$. The inequality $U_{S^{\prime}} \geq U_{M B}\left(P_{S^{\prime}}, P_{S}\right)$ implies that buying all items in $S$ gives a higher utility than buying all except the subset $S^{\prime}$ because

$$
U_{S}+v\left(M-P_{S}\right) \geq U_{S}-U_{S^{\prime}}+v\left(M-P_{S}+P_{S^{\prime}}\right) \Leftrightarrow U_{S^{\prime}} \geq U_{M B}\left(P_{S^{\prime}}, P_{S}\right) .
$$

Since $S^{\prime}$ can be any subset of $S$, we conclude that buying all the items in $S$ is optimal when (10) is satisfied.

\section{Proof of Theorem 1}

We first prove two lemmas which allow us to prove theorem 1 . In the first lemma, we take the set $A$ of active publishers as given and show that there exists a unique candidate equilibrium price vector. The second lemma describes how $A$ is uniquely determined depending on the level of $M$. Therefore, the two lemmas identify a unique equilibrium candidate as a function of $M$. Finally, in the proof of theorem 1, we prove that the candidate is indeed an equilibrium. Let $\underline{j}=\max \{j: j \in A\}$ denote the active publisher with the lowest valued bundle; obviously, $\underline{j}$ depends on $A$ even though we do not emphasize this fact in the notation. Furthermore, let $\underline{A}=A \backslash\{\underline{j}\}$ denote the set of active publishers excluding $\underline{j}$.

Lemma 3 For a given $A^{*}$,

(i) A candidate equilibrium price vector $\mathbf{P}_{A^{*}}{ }^{35}$ satisfying $\pi^{B *}=M$ exists if and only if $\sum_{j \in \underline{A}^{*}} v^{-1}\left(U_{j}-U_{\underline{j}}\right)<M \leq \sum_{j \in A^{*}} v^{-1}\left(U_{j}\right)$; furthermore, $\mathbf{P}_{A^{*}}^{*}$ is unique and satisfies (11):

$$
U_{j}-v\left(P_{j}^{*}\right)=U_{j^{\prime}}-v\left(P_{j^{\prime}}^{*}\right) \geq 0 \quad \text { for any }\left\{j, j^{\prime}\right\} \subseteq A^{*} .
$$

(ii) A candidate equilibrium price vector $\mathbf{P}_{A^{*}}^{*}$ satisfying $\pi^{B *}<M$ exists if and only if $\sum_{j \in A^{*}} v^{-1}\left(U_{j}\right)<M ;$ furthermore, $\mathbf{P}_{A^{*}}^{*}$ is unique and satisfies (12):

$$
U_{j}-U_{M B}\left(P_{j}^{*}, \pi^{B *}\right)=0 \quad \text { for any } j \in A^{*} .
$$

(iii) If $\left(A^{*}, \mathbf{P}_{A^{*}}^{*}\right)$ is an equilibrium of $\Gamma^{B}$, it is necessary that $\sum_{j \in \underline{A}^{*}} v^{-1}\left(U_{j}-U_{\underline{j}}\right)<M$ holds.

\footnotetext{
${ }^{35}$ For clarity we use the notation $\mathbf{P}_{A^{*}}^{*}$ in Appendix instead of $\mathbf{P}^{*}$ which is used in the main text.
} 
Proof of (i): In any equilibrium of $\Gamma^{B}, \mathbf{P}_{A^{*}}^{*}$ is such that $\mathrm{B} j$ is sold for any $j \in A^{*}$; otherwise publisher $j$ will not be active. Since $\pi^{B *}=M$, lemma 2 implies

$$
U_{j} \geq U_{M B}\left(P_{j}^{*}, M\right)=v\left(P_{j}^{*}\right) \quad \text { for any } j \in A^{*}
$$

In order for no publisher to have an incentive to increase the price of his bundle above the price in $\mathbf{P}_{A^{*}}^{*}$, the following condition must be satisfied:

$$
U_{j}-v\left(P_{j}^{*}\right)=U_{j^{\prime}}-v\left(P_{j^{\prime}}^{*}\right) \quad \text { for any } \quad j, j^{\prime} \text { in } A^{*}
$$

If instead $U_{j}-v\left(P_{j}^{*}\right)>U_{j^{\prime}}-v\left(P_{j^{\prime}}^{*}\right)$ for some $j$ and $j^{\prime}$ in $A^{*}$, we can show that publisher $j$ can increase $P_{j}$ slightly from $P_{j}^{*}$ to $P_{j}^{*}+\varepsilon$ and sell his bundle. After $j$ 's price change, the library cannot afford to buy all the bundles in $A^{*}$ because the sum of the prices is larger than $M$. However, lemma 2 and (13) imply that it will drop exactly one bundle. ${ }^{36}$ Given $U_{A^{*}}=\sum_{h \in A^{*}} U_{h}$, the alternative of dropping $\mathrm{B} j$ is suboptimal since it gives the library a smaller payoff with respect to dropping $\mathrm{B} j^{\prime}: U_{A^{*}}-U_{j^{\prime}}+v\left(P_{j^{\prime}}^{*}-\varepsilon\right)>U_{A^{*}}-U_{j}+v\left(P_{j}^{*}\right)$ for a small $\varepsilon$. This establishes that $\mathrm{B} j$ will be purchased and publisher $j$ 's profit increases. Hence, (14) needs to hold. Finally, (13) and (14) imply that (11) holds.

Now we prove that a (unique) $\mathbf{P}_{A^{*}}^{*}$ satisfying $\pi^{B *}=M,(11)$ and $P_{j}^{*}>0$ for all $j \in A^{*}$ exists if and only if $\sum_{j \in \underline{A}^{*}} v^{-1}\left(U_{j}-U_{\underline{j}}\right)<M \leq \sum_{j \in A^{*}} v^{-1}\left(U_{j}\right)$. Use (11) to write $P_{j}$ as a function of $P_{\underline{j}}$ as follows: $P_{j}=v^{-1}\left[\bar{U}_{j}-U_{\underline{j}}+v\left(P_{\underline{j}}\right)\right]$, for any $j \in \underline{A}^{*}$. After combining this with $\pi^{B *}=M$, we obtain:

$$
F\left(P_{\underline{j}}\right) \equiv \sum_{j \in \underline{A}^{*}} v^{-1}\left[U_{j}-U_{\underline{j}}+v\left(P_{\underline{j}}\right)\right]+P_{\underline{j}}-M=0
$$

$F$ is strictly increasing in $P_{\underline{j}}$ and $v^{-1}\left(U_{\underline{j}}\right)$ is the highest value of $P_{\underline{j}}$ consistent with (13). Since $F(0)=\sum_{j \in \underline{A}^{*}} v^{-1}\left(U_{j}-U_{\underline{j}}\right)-M$ and $F\left[v^{-1}\left(U_{\underline{j}}\right)\right]=\sum_{j \in A^{*}} v^{-1}\left(U_{j}\right)-M$, a (unique) solution $P_{\underline{j}}^{*} \in\left(0, v^{-1}\left(U_{\underline{j}}\right)\right]$ to $(15)$ exists if and only if $\sum_{j \in \underline{A}^{*}} v^{-1}\left(U_{j}-U_{\underline{j}}\right)<M \leq$ $\sum_{j \in A^{*}} v^{-1}\left(U_{j}\right)$ is satisfied. Notice that $P_{j}^{*}=v^{-1}\left[U_{j}-U_{\underline{j}}+v\left(P_{\underline{j}}^{*}\right)\right] \geq P_{\underline{j}}^{*}>0$ for any $j \in \underline{A}^{*} \cdot \diamond$

Proof of (ii): Since $\pi^{B *}<M$, lemma 2 implies $U_{j}+v\left(M-\pi^{B *}\right) \geq v\left(M-\pi^{B *}+P_{j}^{*}\right)$ for any $j \in A^{*}$. We prove that if this inequality holds strictly for one $j \in A^{*}$, then publisher $j$ can increase his profit by choosing $P_{j}=P_{j}^{*}+\varepsilon$ with $\varepsilon(>0)$ small. By lemma 2 , if the

\footnotetext{
${ }^{36}$ Suppose that $\mathrm{B} k$ is not purchased for some $k \in A^{*} \backslash\{j\}$. Then, (10) is satisfied for $S=A^{*} \backslash\{k\}$ because (13) implies $U_{h}>U_{M B}\left(P_{h}^{*}, M-P_{k}^{*}+\varepsilon\right)$ for any $h \in A^{*} \backslash\{k, j\}$ and $U_{j}>U_{M B}\left(P_{j}^{*}+\varepsilon, M-P_{k}^{*}+\varepsilon\right)$ for $\varepsilon(>0)$ small enough. If $\mathrm{B} j$ is not purchased, then (10) is satisfied for $S=A^{*} \backslash\{j\}$ because (13) implies $U_{h}>U_{M B}\left(P_{h}^{*}, M-P_{j}^{*}\right)$ for any $h \in A^{*} \backslash\{j\}$.
} 
library does not buy B $j$ then it buys all other bundles because $U_{h}>U_{M B}\left(P_{h}^{*}, \pi^{B *}-P_{j}^{*}\right)$ for any $h \in A^{*} \backslash\{j\}$. Its payoff is then $U_{A^{*}}-U_{j}+v\left(M-\pi^{B *}+P_{j}^{*}\right)$. If the library also buys $\mathrm{B} j$, then its payoff is $U_{A^{*}}+v\left(M-\pi^{B *}-\varepsilon\right)$ and this is larger than $U_{A^{*}}-U_{j}+v\left(M-\pi^{B *}+P_{j}^{*}\right)$ since $U_{j}+v\left(M-\pi^{B *}\right)>v\left(M-\pi^{B *}+P_{j}^{*}\right)$ and $\varepsilon$ is close to $0 .{ }^{37}$ Therefore, (12) needs to be satisfied by $\mathbf{P}_{A^{*}}^{*}$.

Now we prove that a (unique) $\mathbf{P}_{A^{*}}^{*}$ satisfying $\pi^{B *}<M,(12)$ and $P_{j}^{*}>0$ for all $j \in A^{*}$ exists if and only if $\sum_{j \in A^{*}} v^{-1}\left(U_{j}\right)<M$. From (12) we obtain $P_{j}=\pi-M+v^{-1}\left[U_{j}+v(M-\right.$ $\pi)$ ] for all $j \in A^{*}$ and adding it over $j$ yields $\pi=\sum_{j \in A^{*}} v^{-1}\left[U_{j}+v(M-\pi)\right]+\left|A^{*}\right|(\pi-M)$, where $\left|A^{*}\right|$ is the number of publishers in $A^{*}$. Hence, we need to find $\pi \in(0, M)$ such that $G(\pi)=0$, with

$$
G(\pi)=\sum_{j \in A^{*}} v^{-1}\left[U_{j}+v(M-\pi)\right]+\left(\left|A^{*}\right|-1\right) \pi-\left|A^{*}\right| M
$$

Notice that (i) $G$ strictly decreases in $\pi$; (ii) $G(0)>0$ since $U_{j}>0$ for any $j$; (iii) $G(M)=\sum_{j \in A^{*}} v^{-1}\left(U_{j}\right)-M$. Thus, a (unique) solution $\pi^{B *} \in(0, M)$ to (16) exists if and only if $M>\sum_{j \in A^{*}} v^{-1}\left(U_{j}\right)$. Notice that $P_{j}^{*}=\pi^{B *}-M+v^{-1}\left[U_{j}+v\left(M-\pi^{B *}\right)\right]>0$ since $\frac{\partial P_{j}^{*}}{\partial \pi^{B *}}<0$ and $P_{j}^{*}>M-M+v^{-1}\left[U_{j}+v(M-M)\right]=v^{-1}\left(U_{j}\right)>0 . \diamond$

Proof of (iii): (i) and (ii) of lemma 3 show that $M>\sum_{j \in \underline{A}^{*}} v^{-1}\left(U_{j}-U_{\underline{j}}\right)$ is a necessary condition for $\left(A^{*}, \mathbf{P}_{A^{*}}^{*}\right)$ to be an equilibrium of $\Gamma^{B}$.

Next lemma is about determining the set $A^{*}$.

Lemma 4 Suppose that $\left(A^{*}, \mathbf{P}_{A^{*}}^{*}\right)$ is an equilibrium of $\Gamma^{B}$. Then

(i) Publisher 1 is active for any $M>0$. If $\sum_{j=1}^{k-1} v^{-1}\left(U_{j}-U_{k}\right)<M$ for some $k \in$ $\{2, \ldots, N\}$, then $\{1, \ldots, k\} \subseteq A^{*}$.

(ii) If $M \leq v^{-1}\left(U_{1}-U_{2}\right)$, then $A^{*}=\{1\}$. If $\sum_{j=1}^{k-1} v^{-1}\left(U_{j}-U_{k}\right)<M \leq \sum_{j=1}^{k} v^{-1}\left(U_{j}-\right.$ $\left.U_{k+1}\right)$ for some $k \in\{2, \ldots, N-1\}$, then $j \notin A^{*}$ for any $j>k$.

Proof of (i): Suppose that $\sum_{j=1}^{k-1} v^{-1}\left(U_{j}-U_{k}\right)<M$ for some $k \in\{2, \ldots, N\}$ and $h \notin A^{*}$ for some $h \leq k$. We now show that publisher $h$ can make a profit by choosing a suitable $P_{h}$. First, if $\pi^{B *}<M$ it is trivial to see that $\mathrm{B} h$ is purchased at $P_{h}>0$ close to 0 since $U_{h}+v\left(M-\pi^{B *}-P_{h}\right)>v\left(M-\pi^{B *}\right)$ holds.

Consider now $\pi^{B *}=M$. Then, it is enough to prove that the inequality $U_{h}>U_{\underline{j}}-v\left(P_{\underline{j}}^{*}\right)$ holds since we can apply the argument in the proof of lemma 3(i) to show that publisher

\footnotetext{
${ }^{37}$ Notice that we are not proving that the library will buy all the bundles after the increase in the price of $\mathrm{B} j$. We rather prove that the (only) alternative in which $\mathrm{B} j$ is not purchased is suboptimal. Hence, the library will buy $\mathrm{B} j$ but it may not buy $\mathrm{B} j^{\prime}$ if $U_{j^{\prime}}+v\left(M-\pi^{B *}\right)=v\left(M-\pi^{B *}+P_{j^{\prime}}^{*}\right)$.
} 
$h$ can sell his bundle by setting $P_{h}>0$ close to 0 . To show $U_{h}>U_{\underline{j}}-v\left(P_{\underline{j}}^{*}\right)$, we distinguish the case of $\underline{j}>h$ from the case of $\underline{j}<h$. In the case of $\underline{j}>h$, the inequality $U_{h}>U_{\underline{j}}-v\left(P_{\underline{j}}^{*}\right)$ follows simply from $U_{h} \geq U_{\underline{j}}$. In the case of $\underline{j}<h$, we know from lemma 3 that $\mathbf{P}_{A^{*}}^{*}$ satisfies (11) and therefore $P_{\underline{j}}^{*}$ solves (15). The inequality $P_{\underline{j}}^{*}>$ $v^{-1}\left(U_{\underline{j}}-U_{h}\right)$, which is equivalent to $U_{h}>U_{\underline{j}}-v\left(P_{\underline{j}}^{*}\right)$, holds because $F$ is strictly increasing and $F\left(v^{-1}\left(U_{\underline{j}}-U_{h}\right)\right)=\sum_{j \in A^{*}} v^{-1}\left(U_{j}-U_{h}\right)-M$ is strictly negative since $A^{*} \subset\{1, \ldots, k\}$, $\sum_{j=1}^{k-1} v^{-1}\left(U_{j}-U_{k}\right)<M$ (by assumption) and $U_{h} \geq U_{k}$. Notice that if $h=1$, then only the case $\underline{j}>h$ may arise and therefore $1 \in A^{*}$ for any $M>0$. $\diamond$

Proof of (ii): In this proof, let $k=1$ if $M \leq v^{-1}\left(U_{1}-U_{2}\right)$; otherwise $k$ is defined as in the statement of lemma 4(ii). From lemma 4(i) we know that $\{1, \ldots, k\} \subseteq A^{*}$. We below show that a contradiction arises if $\underline{j}>k$. Since, from lemma 3(iii), the inequality $\sum_{j \in \underline{A}^{*}} v^{-1}\left(U_{j}-U_{\underline{j}}\right)<M$ should be satisfied and since we assume $M \leq \sum_{j=1}^{k} v^{-1}\left(U_{j}-\right.$ $\left.U_{k+1}\right)$, it follows that $\sum_{j \in \underline{A}^{*}} v^{-1}\left(U_{j}-U_{\underline{j}}\right)<\sum_{j=1}^{k} v^{-1}\left(U_{j}-U_{k+1}\right)$ must hold. However, the last inequality fails to hold if $\underline{j}>k$ since this implies $\{1, \ldots, k\} \subseteq \underline{A}^{*}$ and $U_{k+1} \geq U_{\underline{j}}$.

\section{Proof of theorem 1}

Proof of (i): Lemmas 4(ii) and 3 imply, respectively, $A^{*}=\{1\}$ and $P_{1}^{*}=M$.

Proof of (ii) Lemmas 4 and 3 imply, respectively, that $A^{*}=\{1, \ldots, k\}$ in any equilibrium and $\mathbf{P}_{A^{*}}^{*}$ is the unique solution to (11). We now prove that $\left(A^{*}, \mathbf{P}_{A^{*}}^{*}\right)$ is indeed an equilibrium of $\Gamma^{B}$ by proving the two following claims.

Claim 1 Publisher $h \in A^{*}$ cannot make a profit larger than $P_{h}^{*}$ given $A=A^{*}$ and $P_{j}=P_{j}^{*}$ for any $j \in A^{*} \backslash\{h\}$.

Proof Let $h \in A^{*}$. We know from lemma 2 that all the bundles in $A^{*}$ are sold if $P_{h} \leq P_{h}^{*}$; we below prove that $\mathrm{B} h$ is not purchased if $P_{h}>P_{h}^{*}$. Clearly, when $P_{h}>P_{h}^{*}$ the library cannot afford to buy all the available bundles. If it buys $\mathrm{B} h$, let $Z \neq \emptyset$ denote the set of the bundles in $A^{*}$ that it does not buy anymore, with $U_{Z} \equiv \sum_{z \in Z} U_{z}$ and $P_{Z}^{*} \equiv \sum_{z \in Z} P_{z}^{*}$. Then, the library's payoff is $U_{A^{*}}-U_{Z}+v\left(P_{Z}^{*}-\left(P_{h}-P_{h}^{*}\right)\right)$, with $U_{A^{*}}=\sum_{z \in A^{*}} U_{z}$. If instead the library buys all the bundles except $\mathrm{B} h$, its payoff is $U_{A^{*}}-U_{h}+v\left(P_{h}^{*}\right)$. We prove that the latter payoff is strictly larger than the former for any $P_{h}>P_{h}^{*}$ by showing that the weak inequality holds at $P_{h}=P_{h}^{*}: v\left(P_{Z}^{*}\right)-v\left(P_{h}^{*}\right) \leq U_{Z}-U_{h}$. Given any $z \in Z$, (11) implies $U_{h}-v\left(P_{h}^{*}\right)=U_{z}-v\left(P_{z}^{*}\right)$ and therefore the latter inequality is equivalent to

$$
v\left(P_{Z}^{*}\right) \leq v\left(P_{z}^{*}\right)+U_{Z}-U_{z}
$$

If $Z \backslash\{z\}=\emptyset$, then (17) trivially holds. If $Z \backslash\{z\} \neq \emptyset$, then (17) holds because $v\left(P_{z}^{*}\right)+$ $U_{Z}-U_{z}=v\left(P_{z}^{*}\right)+\sum_{j \in Z \backslash\{z\}} U_{j} \geq \sum_{j \in Z} v\left(P_{j}^{*}\right)>v\left(P_{Z}^{*}\right)$, where the first inequality comes from (11) and the second one from the strict concavity of $v(\cdot)$. 
Claim 2 Publisher $h \notin A^{*}$ cannot make a positive profit given $A=A^{*}$ and $P_{j}=P_{j}^{*}$ for any $j \in A^{*}$.

Proof Let $h \notin A^{*}$. We below prove that at no price $P_{h}>0$ the bundle $\mathrm{B} h$ will be sold. For this purpose, we first show that $U_{j}-v\left(P_{j}^{*}\right) \geq U_{h}$ for any $j \in A^{*}$. Given $A^{*}=\{1, \ldots, k\}$, we have $\underline{j}=k$ and the solution $P_{k}^{*}$ of equation (15) is weakly smaller than $v^{-1}\left(U_{k}-U_{k+1}\right)$ because $F\left(v^{-1}\left(U_{k}-U_{k+1}\right)\right)=\sum_{j=1}^{k} v^{-1}\left(U_{j}-U_{k+1}\right)-M$ and $\sum_{j=1}^{k} v^{-1}\left(U_{j}-U_{k+1}\right) \geq M$ by assumption. The inequality $P_{k}^{*} \leq v^{-1}\left(U_{k}-U_{k+1}\right)$ is equivalent to $U_{k}-v\left(P_{k}^{*}\right) \geq U_{k+1}$ and this implies, from (11) and $U_{k+1} \geq U_{h}$, that $U_{j}-v\left(P_{j}^{*}\right) \geq U_{h}$ for any $j \in A^{*}$. Using this inequality, we can argue as in the proof of Claim 1 above to show that at no price $P_{h}>0$ the bundle $\mathrm{B} h$ will be sold; hence, it is a best reply for publisher $h$ to be non-active.

Proof of (iii): Lemmas 4(i) and 3(ii) imply, respectively, that $A^{*}=\{1, \ldots, N\}$ and $\mathbf{P}_{A^{*}}^{*}$ is the unique solution to (12). The proof that no publisher $h$ has an incentive to choose $P_{h} \neq P_{h}^{*}$ is very similar to the proof of Claim 1 above, hence it is omitted.

\section{Proof of Proposition 2}

We use $I \subseteq A^{*}$ to represent the set of active publishers which sell their journals independently; $B \equiv A^{*} \backslash I$ is the set of active publishers which bundle their journals. Let $\pi_{j}$ denote the profit of publisher $j, j \in A^{*} ; \pi \equiv \sum_{j \in A^{*}} \pi_{j}$ is the industry profit. Obviously, in any equilibrium $\mathrm{B} j$ is sold for any $j \in B$ and at least a journal of publisher $j$ is sold, for any $j \in I$. Let $R_{j}$ and $Z_{j}$, for $j \in I$, have the same meanings as in the proof of claim 2 of proposition $1 ; R \equiv \cup_{j \in I} R_{j}$ and $Z \equiv \cup_{j \in I} Z_{j}$.

Proof of (i) Suppose that $j \in I$ and the library optimally spends $\pi_{j}(>0)$ on buying some journals of publisher $j$. Then it is still optimal for the library to buy $\mathrm{B} j$ at price $\pi_{j}$ : otherwise, the library would have improved its payoff by not buying any journal from publisher $j$ when his journals were sold independently.

Proof of (ii) We prove the result after proving two claims, which establish that all journals of all publishers in $I$ are sold.

Claim 1 In any equilibrium of $\Gamma$ with $|I| \geq 2$, each journal of each publisher in $I$ has the same price, denoted by $\tilde{p}$, and the library buys all journals; hence, $Z=\emptyset$.

Proof The proof of this claim is the same as the proof of claim 2 in the proof of Proposition 1.

Claim 2 There exists no equilibrium of $\Gamma$ with $|I|=1$ and $Z \neq \emptyset$.

Proof Without loss of generality, let $I=\{1\}$ and suppose that $Z_{1} \neq \emptyset$. Let $p_{1}^{(1)} \equiv$ $\max _{i 1 \in R_{1}}\left\{p_{i 1}\right\}$ be the price of the most expensive journal among the journals of 1 which the library buys. First, notice that if $\pi<M$ then publisher 1 can increase his profit by 
charging a uniform price $p_{1}=\frac{\pi_{1}+\varepsilon}{n_{1}}$ for all his journals, where $\pi_{1}$ is his profit before this deviation. Then all journals of 1 are sold since $u \geq U_{M B}\left(p_{1}^{(1)}, \pi\right)$ and $p_{1}^{(1)}>p_{1}$ imply $u>U_{M B}\left(p_{1}, \pi+\varepsilon\right)$ for $\varepsilon(>0)$ small enough.

Second, when $\pi=M$, we distinguish the case of $U_{j}-v\left(P_{j}\right) \leq u-v\left(p_{1}^{(1)}\right)$ for some $j \in B$ from the case of $U_{j}-v\left(P_{j}\right)>u-v\left(p_{1}^{(1)}\right)$ for all $j \in B$. When $U_{j}-v\left(P_{j}\right) \leq u-v\left(p_{1}^{(1)}\right)$ for some $j \in B, 1$ can increase his profit by choosing a small price $\varepsilon>0$ for one of his journals in $Z_{1}$ - denoted by $i 1$ - and reducing by $\delta$ the price of each journal in $R_{1}$ of which the price is equal to $p_{1}^{(1)}$ in such a way that the sum of prices of journals in $R_{1} \cup\{i 1\}$ is larger by $\alpha>0$ than before. The library cannot afford to buy $i 1$ and all the items it purchased before the deviation, but it will buy all of these items except one, by lemma 2 . Dropping a journal of 1 with price $p_{1}^{(1)}-\delta$ gives payoff $U^{\prime}+v\left(p_{1}^{(1)}-\delta-\alpha\right)$ where $U^{\prime}$ is the total surplus from journals and bundles the library obtains before 1's price changes. Dropping $\mathrm{B} j$ yields $U^{\prime}+u-U_{j}+v\left(P_{j}-\alpha\right)$ which is larger than $U^{\prime}+v\left(p_{1}^{(1)}-\delta-\alpha\right)$ at $\alpha=0$ and therefore also at some $\alpha>0$ by continuity.

Now suppose that $U_{j}-v\left(P_{j}\right)>u-v\left(p_{1}^{(1)}\right)$ for all $j \in B$. Then, a necessary condition for a particular $j \in B$ not to profitably deviate by slightly increasing the price of $\mathrm{B} j$ is that there exists a $t \geq 1$ such that

$$
U^{\prime}-U_{j}+t u+v\left(P_{j}-t \bar{p}\right) \geq U^{\prime}-u+v\left(p_{1}^{(1)}\right)
$$

where $\bar{p}$ is the average price of the $t$ cheapest journals in $Z_{1}$. We prove now that a profitable deviation for publisher 1 is to bundle all his journals at a price $P_{1}=\pi_{1}+\varepsilon>\pi_{1}$. Once again, only one bundle will be dropped. The library's payoff from dropping $\mathrm{B} j$ is $U^{\prime}+\left|Z_{1}\right| u-U_{j}+v\left(P_{j}-\varepsilon\right)$ while the payoff from dropping B1 is $U^{\prime}-\left|R_{1}\right| u+v\left(\pi_{1}\right)$. The latter payoff is smaller than the former because now we prove that $U_{1}+v\left(P_{j}-\varepsilon\right)>U_{j}+v\left(\pi_{1}\right)$ for a small $\varepsilon>0$. Inequality (18) is equivalent to $t u+v\left(P_{j}-t \bar{p}\right) \geq U_{j}-u+v\left(p_{1}^{(1)}\right)$; hence $\left|Z_{1}\right| \geq t$ implies $U_{1}+v\left(P_{j}-\varepsilon\right) \geq\left|R_{1}\right| u+v\left(P_{j}-\varepsilon\right)+U_{j}-u+v\left(p_{1}^{(1)}\right)-v\left(P_{j}-t \bar{p}\right)$ and this right hand side is larger than $U_{j}+v\left(\pi_{1}\right)$, or $\left(\left|R_{1}\right|-1\right) u+v\left(P_{j}-\varepsilon\right)+v\left(p_{1}^{(1)}\right)-v\left(P_{j}-t \bar{p}\right) \geq v\left(\pi_{1}\right)$, because of the following argument. First notice that $v\left(P_{j}-\varepsilon\right)>v\left(P_{j}-t \bar{p}\right)$ for small $\varepsilon$. Second, if $\left|R_{1}\right|=1$ then $p_{1}^{(1)}=\pi_{1}$ and the result is straightforward. If $\left|R_{1}\right| \geq 2$, then $\left(\left|R_{1}\right|-1\right) u+v\left(p_{1}^{(1)}\right) \geq\left|R_{1}\right| v\left(p_{1}^{(1)}\right)>v\left(\left|R_{1}\right| p_{1}^{(1)}\right) \geq v\left(\pi_{1}\right) . \diamond$

By claims 1 and 2, in the rest of the proof we assume that all journals of all publishers in $I$ are sold and $|I| \geq 2$.

Consider first the case of $\pi=M$. Then the equality $u_{s}-v\left(p_{s}\right)=u_{s^{\prime}}-v\left(p_{s^{\prime}}\right)=b \geq 0$ must hold for any pair of items $s$ and $s^{\prime}$ (journal or bundles). Precisely, $b \geq 0$ by lemma 2 and if $u_{s}-v\left(p_{s}\right)>u_{s^{\prime}}-v\left(p_{s^{\prime}}\right)$, then the publisher of item $s$ can increase his profit by 
increasing slightly the price of $s$. This statement is proved by arguing exactly like in the proof of lemma 3(i) if $s$ is a bundle. If instead $s$ is a journal, then $s^{\prime}$ is a bundle since $p_{i j}=\tilde{p}$ for any journal $i j$ (by claim 1 in this proof) and therefore $u_{i j}-v\left(p_{i j}\right)>u_{s^{\prime}}-v\left(p_{s^{\prime}}\right)$ for any $i j$. The argument of the proof of lemma 3(i) shows that a publisher in $I$ still sells all his journals if he increases the price of one of them to $\tilde{p}+\varepsilon$ with $\varepsilon>0$ and small. Now let publisher $j \in I$ bundle his $n_{j} \geq 2$ journals at price $\pi_{j}+\varepsilon$ with $\varepsilon(>0)$ small enough; the library has not enough money to buy all the available items, but after arguing like in the proof of lemma 3(i) we see that it will purchase all items except one. The payoff from dropping $\mathrm{B} j$ is $U-u n_{j}+v\left(\pi_{j}\right)$ and the payoff from not buying a different item $s$ is $U-u_{s}+v\left(p_{s}-\varepsilon\right)$. Since $\pi_{j}=n_{j} \tilde{p}$ and $n_{j} u-v\left(n_{j} \tilde{p}\right)>n_{j} b \geq b$, the inequality $n_{j} u-v\left(\pi_{j}\right)>u_{s}-v\left(p_{s}-\varepsilon\right)$ holds for a small $\varepsilon$. Therefore, the library prefers dropping item $s$ to dropping $\mathrm{B} j$.

Consider now the case in which $\pi<M$. Then we can prove that $u_{s}=U_{M B}\left(p_{s}, \pi\right)$ for any item $s$. Precisely, $u_{s} \geq U_{M B}\left(p_{s}, \pi\right)$ for any $s$ by lemma 2 and if $u_{s}>U_{M B}\left(p_{s}, \pi\right)$ for some $s$, then the publisher of item $s$ can increase his profit by increasing slightly the price of $s$. The proof of this result mimics the arguments given above for the case of $\pi=M$ and the proof of lemma 3(ii). Now let publisher $j$ bundle his $n_{j} \geq 2$ journals at price $P_{j}=\pi_{j}+\varepsilon$ with $\varepsilon(>0)$ small enough. We prove that $\mathrm{B} j$ is sold and therefore publisher $j$ increases his profit. Lemma 2, once again, implies that at most one item is not purchased. Suppose by contradiction that it is $\mathrm{B} j$; then, the library's payoff is $U-n_{j} u+v\left(M-\pi+\pi_{j}\right)$. If $\mathrm{B} j$ is added to the other items, the payoff increases by

$$
n_{j} u-U_{M B}\left(\pi_{j}+\varepsilon, \pi+\varepsilon\right)
$$

From $u=U_{M B}(\tilde{p}, \pi), \tilde{p} n_{j}=\pi_{j}$ and lemma $1(\mathrm{ii})$, we find $n_{j} u>U_{M B}\left(\pi_{j}, \pi\right)$. Hence, $(19)$ is positive at $\varepsilon=0$ and also for a small $\varepsilon>0$.

\section{Proof of Proposition 4}

Suppose that all publishers are active before the merger. Without loss of generality, we assume that publisher 1 merges with publisher 2 . Let $P_{j}^{B M *}$ and $P_{j}^{A M *}$ denote the prices before the merger and after the merger, respectively, of $\mathrm{B} j, j=1, \ldots, N ; P_{1 \& 2}^{*}$ is the price charged by publisher $1 \& 2$ after the merger. Consider the case in which $\sum_{j=1}^{N} v^{-1}\left(U_{j}\right)<M$, so that $\pi^{B M *}<M$ and assume that $v^{-1}\left(U_{1}+U_{2}\right)+\sum_{j=3}^{N} v^{-1}\left(U_{j}\right)<M$; this implies $\pi^{A M *}<M$. To prove that $\pi^{A M *}>\pi^{B M *}$, we suppose by contradiction that $\pi^{A M *} \leq \pi^{B M *}$. Condition (4) implies

$$
\begin{aligned}
& U_{1}+U_{2}=U_{M B}\left(P_{1}^{B M *}, \pi^{B M *}\right)+U_{M B}\left(P_{2}^{B M *}, \pi^{B M *}\right) \\
& U_{1}+U_{2}=U_{M B}\left(P_{1 \& 2}^{*}, \pi^{A M *}\right)
\end{aligned}
$$


Since $U_{M B}\left(P_{1}^{B M *}, \pi^{B M *}\right)+U_{M B}\left(P_{2}^{B M *}, \pi^{B M *}\right)>U_{M B}\left(P_{1}^{B M *}+P_{2}^{B M *}, \pi^{B M *}\right)$, we have $U_{M B}\left(P_{1 \& 2}^{*}, \pi^{A M *}\right)>U_{M B}\left(P_{1}^{B M *}+P_{2}^{B M *}, \pi^{B M *}\right)$. This inequality and $\pi^{A M *} \leq \pi^{B M *}$ imply $P_{1 \& 2}^{*}>P_{1}^{B M *}+P_{2}^{B M *}$. Furthermore, (4) for $j=3, \ldots, N$ implies

$$
\frac{d P_{j}}{d \pi}=1-\frac{v^{\prime}(M-\pi)}{v^{\prime}\left(M-\pi+P_{j}\right)}<0
$$

which says that the merger (weakly) increases the profit of any non-merged publisher. Since the merger increases each publisher's profit, it contradicts the assumption $\pi^{A M *} \leq$ $\pi^{B M *}$. Therefore, we must have $\pi^{A M *}>\pi^{B M *}$; this implies that the profit of publisher $j(j=3, . ., N)$ is reduced because of $(22)$ and hence the profit of publisher $1 \& 2$ is larger than $P_{1}^{B M *}+P_{2}^{B M *}$.

In the case that $\pi^{A M *}=M$, then it is obvious that $\pi^{A M *}>\pi^{B M *}$. Then (22) implies again that the profit of publisher $j(j=3, . ., N)$ is reduced and, as a consequence, the profit of publisher $1 \& 2$ is larger than $P_{1}^{B M *}+P_{2}^{B M *}$.

The result can be similarly proved when $\sum_{j=1}^{k-1} v^{-1}\left(u_{j}-u_{k}\right)<M \leq \sum_{j=1}^{k} v^{-1}\left(u_{j}-u_{k+1}\right)$ for some $k \geq 3$, so that $A^{*}=\{1, \ldots, k\}$ and $\pi^{B M *}=M$.

\section{Proof of Proposition 5(ii)}

Let $P_{j}^{k}$ denote the equilibrium price for $\mathrm{B} j$ in the case that publisher $k$ wins the auction and bundles the new journal with all his existing journals. Furthermore, let $\pi^{B k} \equiv$ $\sum_{j=1}^{N} P_{j}^{k}$.

Claim $1 \pi^{B 1} \geq \pi^{B 2} \geq \ldots \geq \pi^{B N}$ : the industry profit increases (weakly) more as the new journal is integrated to a larger bundle.

Proof In order to prove that $\pi^{B j} \geq \pi^{B k}$ whenever $j \leq k$, notice that the result is straightforward if $\pi^{B k}=M$ since $U_{j} \geq U_{k}$ and $M \leq v^{-1}\left(U_{1}\right)+\ldots+v^{-1}\left(U_{k}+u\right)+\ldots+$ $v^{-1}\left(U_{N}\right)$ imply $M \leq v^{-1}\left(U_{1}\right)+\ldots+v^{-1}\left(U_{j}+u\right)+\ldots+v^{-1}\left(U_{N}\right)$; therefore, $\pi^{B j}=M$. If instead $\pi^{B k}<M$, then the result is still obvious if $\pi^{B j}=M$. Hence, we need to deal with the case of $\pi^{B k}<M$ and $\pi^{B j}<M$. Without loss of generality, we show that $\pi^{B 1}>\pi^{B 2}$ when $M>\pi^{B 1}$ and $M>\pi^{B 2}$. Suppose that $\pi^{B 1} \leq \pi^{B 2}$. Then lemma 1(i) and (4) imply $P_{h}^{1} \geq P_{h}^{2}$ for all $h \geq 3$; we now prove that $P_{1}^{1}+P_{2}^{1}>P_{1}^{2}+P_{2}^{2}$ to get a contradiction. Notice that $P_{1}^{1}$ and $P_{2}^{1}$ are such that

$$
U_{M B}\left(P_{1}^{1}, \pi^{B 1}\right)=U_{1}+u ; \quad U_{M B}\left(P_{2}^{1}, \pi^{B 1}\right)=U_{2} ;
$$

Define $f_{1}(\pi)$ and $f_{2}(\pi)$ as follows: ${ }^{38}$

$$
U_{M B}\left(f_{1}(\pi), \pi\right)=U_{1} ; \quad U_{M B}\left(f_{2}(\pi), \pi\right)=U_{2}+u .
$$

\footnotetext{
${ }^{38}$ The logic of the proof here mimics the ideas of the proof of proposition 3.
} 
We now prove that $f_{1}\left(\pi^{B 1}\right)$ and $f_{2}\left(\pi^{B 1}\right)$ satisfy $P_{1}^{1}-f_{1}\left(\pi^{B 1}\right)>f_{2}\left(\pi^{B 1}\right)-P_{2}^{1}$; since $v^{\prime}$ is strictly decreasing, $u=U_{M B}\left(P_{1}^{1}, \pi^{B 1}\right)-U_{M B}\left(f_{1}\left(\pi^{B 1}\right), \pi^{B 1}\right)=\int_{f_{1}\left(\pi^{B 1}\right)}^{P_{1}^{1}} v^{\prime}\left(M-\pi^{B 1}+z\right) d z$, $u=U_{M B}\left(f_{2}\left(\pi^{B 1}\right), \pi^{B 1}\right)-U_{M B}\left(P_{2}^{1}, \pi^{B 1}\right)=\int_{P_{2}^{1}}^{f_{2}\left(\pi^{B 1}\right)} v^{\prime}\left(M-\pi^{B 1}+z\right) d z$ and the inequality $f_{1}\left(\pi^{B 1}\right)>P_{2}^{1}$ imply that $P_{1}^{1}-f_{1}\left(\pi^{B 1}\right)>f_{2}\left(\pi^{B 1}\right)-P_{2}^{1}$. Hence, $P_{1}^{1}+P_{2}^{1}>f_{1}\left(\pi^{B 1}\right)+f_{2}\left(\pi^{B 1}\right)$ and finally $f_{1}\left(\pi^{B 1}\right)+f_{2}\left(\pi^{B 1}\right) \geq f_{1}\left(\pi^{B 2}\right)+f_{2}\left(\pi^{B 2}\right)=P_{1}^{2}+P_{2}^{2}$ because $f_{1}$ and $f_{2}$ are decreasing. This gives $P_{1}^{1}+P_{2}^{1}>P_{1}^{2}+P_{2}^{2}$ and the contradiction.

Claim 2 If $N=2$, each publisher has a weakly dominant bid; the dominant bids are such that $b_{1}>b_{2}$ if $\pi^{B 2}<M$, while $b_{1}=b_{2}$ if $\pi^{B 2}=M$.

Proof Suppose that $N=2$. Then, publisher $j$ 's (unique) weakly dominant strategy is $b_{j}=P_{j}^{j}-P_{j}^{k}$ for $j=1,2, k \neq j$. Since

$$
b_{1}-b_{2}=\pi^{B 1}-\pi^{B 2}
$$

we infer that $b_{1}>b_{2}$ if $\pi^{B 2}<M$ because this implies $\pi^{B 1}>\pi^{B 2} ; b_{1}=b_{2}$ if $\pi^{B 2}=M$ because this implies $\pi^{B 1}=\pi^{B 2} . \diamond$

Claim 3 If $N \geq 3$, then $P_{j}^{1}<P_{j}^{h}$ for any $h \notin\{1, j\}$.

Proof Notice that if $\pi^{B h}<M$, then $\pi^{B 1}>\pi^{B h}$ and pecuniary externalities (i.e. (3) or (4)) imply $P_{j}^{1}<P_{j}^{h}$. If instead $\pi^{B h}=M$, then $\pi^{B 1}=\pi^{B h}$ and we prove $P_{j}^{1}<P_{j}^{h}$ as follows. Suppose (without loss of generality, but only to simplify notation), that $\underline{j}=N$ and $h \neq N$. If 1 wins the journal, then $P_{N}^{1}$ solves (15) in the proof of theorem 1 :

$$
v^{-1}\left[U_{1}+u-U_{N}+v\left(P_{N}\right)\right]+\ldots+v^{-1}\left[U_{h}-U_{N}+v\left(P_{N}\right)\right]+\ldots+P_{N}=M
$$

If $h$ wins the journal, then $P_{N}^{h}$ solves

$$
v^{-1}\left[U_{1}-U_{N}+v\left(P_{N}\right)\right]+\ldots+v^{-1}\left[U_{h}+u-U_{N}+v\left(P_{N}\right)\right]+\ldots+P_{N}=M
$$

For a given $P_{N}$, the left hand side in (23) is larger than the left hand side in (24) since $v^{-1}\left[U_{1}+u-U_{N}+v\left(P_{N}\right)\right]-v^{-1}\left[U_{1}-U_{N}+v\left(P_{N}\right)\right]>v^{-1}\left[U_{h}+u-U_{N}+v\left(P_{N}\right)\right]-v^{-1}\left[U_{h}-U_{N}+v\left(P_{N}\right)\right]$

because $U_{1}>U_{h}$ and $v^{-1}$ is convex. Let $P_{N}^{1}$ satisfy (23) and $P_{N}^{h}$ satisfy (24). Given (25), the left hand side of (23) at $P_{N}=P_{N}^{h}$ is larger than $M$; hence $P_{N}^{1}<P_{N}^{h}$. From $P_{j}=v^{-1}\left[U_{j}-U_{N}+v\left(P_{N}\right)\right]$ we get $P_{j}^{1}<P_{j}^{h}$ for all $h \notin\{1, j\}$.

Claim 4 If $N \geq 3$, then $b_{1}>b_{j}$ for any $j \neq 1$ in any undominated equilibrium.

Proof We first prove that for publisher $j \geq 2$ any bid larger than $P_{j}^{j}-P_{j}^{1}$ is weakly dominated by $\hat{b}_{j}=P_{j}^{j}-P_{j}^{1}$ (hence, in any undominated equilibrium publisher $j$ bids $\hat{b}_{j}$ or less). The difference between the profit upon winning the auction and the one 
upon losing it for publisher $j$ is $P_{j}^{j}-P_{j}^{h}$, when publisher $h \neq j$ is the winner. Since $P_{j}^{j}-P_{j}^{h} \leq P_{j}^{j}-P_{j}^{1}$ for any $h \neq j$ by claim 3 , it follows that the difference for publisher $j$ between a bid $b_{j}\left(>\hat{b}_{j}\right)$ and the bid $\hat{b}_{j}$ is that the first bid makes $j$ win also in cases in which he has to pay a price larger than $\hat{b}_{j}$; but in these cases $j$ prefers losing the auction to winning it.

Now suppose that publisher $h$ makes the highest bid among publishers different from 1 , and that he bids $b_{h}$. If $P_{1}^{1}-b_{h}>P_{1}^{h}$, then 1 is happier when he wins the auction at price $b_{h}$ than when he loses it and he can win it at price $b_{h}$ by bidding any number larger than $b_{h}$. Therefore, if 1 is not winning the journal it is necessary that $b_{h} \geq P_{1}^{1}-P_{1}^{h}$. However, we have proved above that any bid larger than $\hat{b}_{h}=P_{h}^{h}-P_{h}^{1}$ is weakly dominated for bidder $h$. We show that $P_{1}^{1}-P_{1}^{h}>\hat{b}_{h}$, which implies that 1 does not win the auction only if some other publisher is playing a weakly dominated strategy. Claim 3 and the inequality $\pi^{B 1} \geq \pi^{B h}$ imply $P_{1}^{1}-P_{1}^{h}>\hat{b}_{h}$.

\section{Proof of Theorem 2}

Proof of theorem 2(i): Consider first the case of $M>\sum_{i=1}^{n} v^{-1}\left(u_{i 1}\right)$. Then we know from corollary 3 that the industry profit $\pi^{I *}$ under the $n$-publisher- $n$-journal setting is smaller than $M$. We prove by contradiction that the monopolist's profit is smaller than $\pi^{I *}$ if he chooses prices different from $\mathbf{p}^{*}$. Suppose that the monopolist can realize a profit $\pi \geq \pi^{I *}$ with $\mathbf{p} \neq \mathbf{p}^{*}$. This implies that, among the journals sold, there must be at least a journal $i 1$ of which the price $p_{i 1}$ is strictly higher than $p_{1 i}^{*}$, the price of journal $1 i$ in the $n$-publisher- $n$-journal setting. Then, we have the contradiction

$$
u_{i 1}=u_{1 i}=U_{M B}\left(p_{1 i}^{*}, \pi^{I *}\right)<U_{M B}\left(p_{i 1}, \pi\right)
$$

given that $\pi \geq \pi^{I *}, p_{i 1}>p_{1 i}^{*}$ and lemma 1 .

If $M \leq \sum_{i=1}^{n} v^{-1}\left(u_{i 1}\right)$, the monopolist can obtain profit $M$ by choosing the prices $\mathbf{p}^{*}$ as under the $n$-publisher- $n$-journal setting because they induce the library to buy all the journals $11, \ldots, n 1$ by lemma 2 .

\section{Proof of theorem 2(ii)(a):}

In this proof, let $A_{J}^{*}$ denote the set of active journals; $i j$ is the journal with the lowest value in $A_{J}^{*}$ and $\underline{A}_{J}^{*} \equiv A_{J}^{*} \backslash\{\underline{i j}\}$. The existence of a unique equilibrium candidate is established by proving the following two lemmas, which parallel lemmas 3 and 4 in the proof of theorem 1 . Lemma 5 shows that given $A_{J}^{*}$, there exists a unique candidate equilibrium price vector and lemma 6 proves that given $M$, there is a unique $A_{J}^{*}$. 
Lemma 5 (i) For a given $A_{J}^{*}$,

(i) A candidate equilibrium price vector $\mathbf{p}_{A_{J}^{*}}^{*}$ satisfying $\pi^{I *}=M$ exists if and only if $\sum_{i j \in \underline{A}_{J}^{*}} v^{-1}\left(u_{i j}-u_{\underline{i j}}\right)<M \leq \sum_{i j \in A_{J}^{*}} v^{-1}\left(u_{i j}\right)$; furthermore, $\mathbf{p}_{A_{J}^{*}}^{*}$ is unique and satisfies (26):

$$
u_{i j}-v\left(p_{i j}^{*}\right)=u_{i^{\prime} j^{\prime}}-v\left(p_{i^{\prime} j^{\prime}}^{*}\right) \geq 0 \quad \text { for any }\left\{i j, i^{\prime} j^{\prime}\right\} \subseteq A_{J}^{*} .
$$

(ii) A candidate equilibrium price vector $\mathbf{p}_{A_{J}^{*}}^{*}$ satisfying $\pi^{I *}<M$ exists if and only if $\sum_{i j \in A_{J}^{*}} v^{-1}\left(u_{i j}\right)<M$; furthermore, $\mathbf{p}_{A_{J}^{*}}^{*}$ is unique and satisfies (27):

$$
u_{i j}-U_{M B}\left(p_{i j}^{*}, \pi\right)=0 \quad \text { for any } i j \in A_{J}^{*} .
$$

(iii) If $\left(A_{J}^{*}, \mathbf{p}_{A_{J}^{*}}^{*}\right)$ is an equilibrium of $\Gamma^{I}$, it is necessary that $\sum_{i j \in \underline{A}_{J}^{*}} v^{-1}\left(u_{i j}-u_{\underline{i j}}\right)<M$ holds.

Proof of (i): In any equilibrium of $\Gamma^{I}, \mathbf{p}_{A_{J}^{*}}^{*}$ is such that each active journal is sold. Since $\pi^{I *}=M$, lemma 2 implies $u_{i j} \geq v\left(p_{i j}^{*}\right)$ for any $i j \in A_{J}^{*}$. Let $b_{i j}=u_{i j}-v\left(p_{i j}^{*}\right)$ for any $i j \in A_{J}^{*}$ and $b=\min _{i j \in A_{J}^{*}}\left\{b_{i j}\right\} \geq 0$; we prove that if $b_{i j}>b$ for some $i j \in A_{J}^{*}$, then there exists a profitable deviation for publisher $j$. Suppose without loss of generality that $j=1$ and that $b_{11}>b, b_{i 1}=b$ for any other $i 1 \in A_{J}^{*}$. Let publisher 1 increase slightly the price of journal 11 to $p_{11}^{\prime}$ and reduce $p_{i 1}$ slightly to $p_{i 1}^{\prime}$ for any other $i 1 \in A_{J}^{*}$ in such a way that the sum of prices of his journals in $A_{J}^{*}$ does not change. Then, $b_{i 1}^{\prime}=u_{i 1}-v\left(p_{i 1}^{\prime}\right)>b$ for all $i 1 \in A_{J}^{*}$ and all active journals are still purchased by lemma 2 . Now let publisher 1 increase the price of journal 11 to $p_{11}^{\prime}+\varepsilon$ with $\varepsilon>0$ and small. We prove that all active journals of 1 are still purchased and therefore his profit increases. Now the library cannot afford to buy all the active journals, but it can afford to (and is willing to, by lemma 2) purchase all active journals except one. Given $U_{A_{J}^{*}}=\sum_{i j \in A_{J}^{*}} u_{i j}$, the library's payoff if it drops journal 11 is $U_{A_{J}^{*}}-\left[u_{11}-v\left(p_{11}^{\prime}\right)\right]$; if it drops $i 1 \in A_{J}^{*} \backslash\{11\}$, the payoff is $U_{A_{J}^{*}}-\left[u_{i 1}-v\left(p_{i 1}^{\prime}-\varepsilon\right)\right]$; if it eliminates a journal $i j(j \neq 1)$ such that $b_{i j}=b$, the payoff is $U_{A_{J}^{*}}-u_{i j}+v\left(p_{i j}^{*}-\varepsilon\right)=U_{A_{J}^{*}}-b-\left[v\left(p_{i j}^{*}\right)-v\left(p_{i j}^{*}-\varepsilon\right)\right]$. Since $u_{i 1}-v\left(p_{i 1}^{\prime}\right)>b$ for any $i 1 \in A_{J}^{*}$, for a small $\varepsilon$ it is better to drop journal $i j$ rather than some journal $i 1 \in A_{J}^{*}$. In this way we infer that $u_{i j}-v\left(p_{i j}^{*}\right)=b$ for any active journal and (26) holds. The proof that there exists a (unique) $\mathbf{p}_{A_{J}^{*}}^{*}$ satisfying $(26), \pi^{I *}=M$ and $p_{i j}^{*}>0$ for any ij $\in A_{J}^{*}$ if and only if $\sum_{i j \in \underline{A}_{J}^{*}} v^{-1}\left(u_{i j}-u_{i j}\right)<M \leq \sum_{i j \in A_{J}^{*}} v^{-1}\left(u_{i j}\right)$ mimics closely the proof of lemma 3(i) and is omitted.

Proofs of (ii)-(iii): These proofs are omitted since they are very similar to the proofs of lemma 3(ii)-(iii) and of (i) above. $\diamond$

In the rest of the proof, $u^{(k)}$ is the value of the journal with the $k$-highest value; hence, $u^{(1)} \geq \ldots \geq u^{(n)}$. 
Lemma 6 Suppose that $\left(A_{J}^{*}, \mathbf{p}_{A_{J}^{*}}^{*}\right)$ is an equilibrium of $\Gamma^{I}$. Then

(i) The journal with the highest value is active for any $M>0$. If $\sum_{j=1}^{k-1} v^{-1}\left(u^{(j)}-u^{(k)}\right)<$ $M$ for some $k \in\{2, \ldots, n\}$, then $A_{J}^{*}$ includes the $k$ journals with the highest values.

(ii) If $M \leq v^{-1}\left(u^{(1)}-u^{(2)}\right)$, then $A_{J}^{*}$ includes only the highest value journal. If $\sum_{j=1}^{k-1} v^{-1}\left(u^{(j)}-\right.$ $\left.u^{(k)}\right)<M \leq \sum_{j=1}^{k} v^{-1}\left(u^{(j)}-u^{(k+1)}\right)$ for some $k \in\{2, \ldots, n-1\}$, then $A_{J}^{*}$ does not include any journal with value smaller than $u^{(k)}$.

Proof of (i): Suppose that $\sum_{j=1}^{k-1} v^{-1}\left(u^{(j)}-u^{(k)}\right)<M$ for some $k \in\{2, \ldots, n\}$ and a journal with value $u^{(h)}\left(\geq u^{(k)}\right)$ is not active. Then, the publisher of this journal can increase his profit. If $\pi<M$, he can make the journal active at a small price and decrease the price of all his other active journals such that the sum of prices of his journals in $A_{J}^{*}$ is slightly larger than his previous profit; then, arguments very similar to those in the proof of lemma 3(ii) show that all his journals will be sold. Thus, his profit will increase. For the setting with $\pi=M$, we can prove that $u^{(h)}>u_{i j}-v\left(p_{i j}^{*}\right)$ for any $i j \in A_{J}^{*}$ by arguing as in the proof of lemma 4(i). Also in this case the publisher of the journal with value $u^{(h)}$ can increase his profit by pricing it close to 0 and decreasing the prices of all his other journals in $A_{J}^{*}$, in a way that the sum of prices is slightly larger than his previous profit. The details are very similar to those in the proof of lemma $5(\mathrm{i}) . \diamond$

Proof of (ii): In this proof, let $k=1$ if $M<v^{-1}\left(u^{(1)}-u^{(2)}\right)$; otherwise $k$ is defined as in the statement of lemma 6(ii). From lemma 6(i) we know that the $k$ journals with the highest values are active; by arguing as in the proof of lemma 4(ii) we find a contradiction if a journal with value smaller than $u^{(k)}$ is active. $\diamond$

\section{Back to the Proof of theorem 2(ii)(a)}

By combining lemmas 5 and 6 we infer that if $\left(A_{J}^{*}, \mathbf{p}_{A_{J}^{*}}^{*}\right)$ is an equilibrium of $\Gamma^{I}$, then $A_{J}^{*}$ is equal to the set of active publishers and $\mathbf{p}_{A_{J}^{*}}^{*}$ is the price vector which is found in the unique equilibrium for the $n$-publisher- $n$-journal environment, described by corollary 3. Hence, there exists a unique candidate equilibrium for the oligopoly setting and it is equal to the equilibrium under the minimal industry concentration.

\section{Proof of theorem 2(ii)(b):}

Let $\mathbf{u} \equiv\left(u_{11}, \ldots, u_{n_{1} 1}, \ldots, u_{1 N}, \ldots, u_{n_{N} N}\right) \in \mathbb{R}_{++}^{n}$ be the vector of the values of the single journals. Given $u>0$, let $\mathbf{u}_{u} \equiv(u, \ldots, u) \in \mathbb{R}_{++}^{n}$ represent the vector of values when journals are homogeneous such that $u_{i j}=u$ for all $i j$. We prove that if $\mathbf{u}$ is close to $\mathbf{u}_{u}$ for some $u>0$, then the unique candidate equilibrium $\left(A_{J}^{*}, \mathbf{p}_{A_{J}^{*}}^{*}\right)$ for $\Gamma^{I}$ determined by corollary 3 is indeed an equilibrium of $\Gamma^{I}$. First notice that, given any $M>0$, the inequality $M>\sum_{h=1}^{n} v^{-1}\left(u^{(h)}-u^{(n)}\right)$ is satisfied if journals are sufficiently homogeneous; this implies that all the journals are sold in the unique candidate equilibrium or, equivalently, that 
$A_{J}^{*}=\tilde{A} \equiv\{1, \ldots, n\}$ and $\mathbf{p}_{A_{J}^{*}}^{*}=\left(p_{11}^{*}, \ldots, p_{n_{1} 1}^{*}, \ldots, p_{1 N}^{*}, \ldots, p_{n_{N} N}^{*}\right) \in \mathbb{R}_{++}^{n}$. For expositional simplicity, we use $\mathbf{p}^{*}$ instead of $\mathbf{p}_{A_{J}^{*}}^{*}$, since $A_{J}^{*}=\tilde{A}$ in all this proof; it should be clear that $\mathbf{p}^{*}$ depends on $\mathbf{u}$ even though the notation does not emphasize this fact. We use $\mathbf{p}_{j}^{*}$ $\left(\mathbf{p}_{-j}^{*}\right)$ to denote the prices in $\mathbf{p}^{*}$ of the journals owned (not owned) by publisher $j$.

Given $u>0$, we now prove that there exists $\delta>0$ such that if the distance $d\left(\mathbf{u}, \mathbf{u}_{u}\right)$ between $\mathbf{u}$ and $\mathbf{u}_{u}$ is smaller than $\delta$ (i.e. if journals are approximately homogeneous), then $\left(\tilde{A}, \mathbf{p}^{*}\right)$ is an equilibrium of $\Gamma^{I}$. Suppose by contradiction that this statement is false. Then, for $t=1,2, \ldots$ there exist (i) $\mathbf{u}^{t} \in \mathbb{R}_{++}^{n}$ such that $d\left(\mathbf{u}^{t}, \mathbf{u}_{u}\right)<\frac{1}{t}$; (ii) the unique candidate equilibrium $\left(\tilde{A}, \mathbf{p}^{*}\right)$ given $\mathbf{u}^{t}$, determined by corollary 3 ; (iii) a publisher $j(t)$, a set of active journals $A_{j(t)}^{t} \subseteq\left\{1 j(t), 2 j(t), \ldots, n_{j(t)} j(t)\right\}$ for him and prices $\mathbf{p}_{j(t)}^{t} \in \mathbb{R}_{++}^{\left|A_{j(t)}^{t}\right|}$ for his active journals such that, given that the other publishers make all their journals active and choose prices $\mathbf{p}_{-j(t)}^{* t}$, the library buys all the journals in $A_{j(t)}^{t}$ and $j(t)$ makes a profit $\sum_{i j(t) \in A_{j(t)}^{t}} p_{i j(t)}^{t}$ which is larger than his profit $\sum_{i=1}^{n_{j(t)}} p_{i j(t)}^{* t}$ if he makes all his journals active with prices $\mathbf{p}_{j(t)}^{* t}$. The latter fact requires that, given $A_{j(t)}^{t}$ and prices $\mathbf{p}^{t} \equiv\left(\mathbf{p}_{j(t)}^{t}, \mathbf{p}_{-j(t)}^{* t}\right)$, the library does not buy all the journals of the other publishers. ${ }^{39}$ Let $Q^{t}$ be the set of all the possible combinations of journals the library can afford to buy given $\left(A_{j(t)}^{t}, \mathbf{p}^{t}\right)$ and let $S_{-j(t)}^{t} \subset \bar{S}_{-j(t)} \equiv\left\{i h: i=1, \ldots, n_{h}\right.$ and $\left.h \neq j(t)\right\}$ be the set of the journals of publishers different from $j(t)$ that the library buys to maximize its own payoff. Notice that, by definition, $\left(A_{j(t)}^{t} \cup S_{-j(t)}^{t}\right) \in Q^{t}$ for any $t$.

Since there are finitely many publishers and journals, there exists a subsequence of the original sequence $\left\{\mathbf{u}^{t}\right\}_{t=1}^{+\infty}$ along which $j(t), S_{-j(t)}^{t}, A_{j(t)}^{t}$ and $Q^{t}$ are all constant. Without loss of generality, we assume that (i) the subsequence is the original sequence: $j(t)=j$, $S_{-j(t)}^{t}=S_{-j}, A_{j(t)}^{t}=A_{j}$ and $Q^{t}=Q$ for all $t$; (ii) $j=1$. Let $U_{A_{1}}^{t}=\sum_{i 1 \in A_{1}} u_{i 1}^{t}$, $\pi_{A_{1}}^{t}=\sum_{i 1 \in A_{1}} p_{i 1}^{t}, U_{S_{-1}}^{t}=\sum_{i j \in S_{-1}} u_{i j}^{t}$ and $\pi_{S_{-1}}^{* t}=\sum_{i j \in S_{-1}} p_{i j}^{* t}$. Then, for any $t$, the library's payoff from buying the journals in $A_{1} \cup S_{-1}$ is $U_{A_{1}}^{t}+U_{S_{-1}}^{t}+v\left(M-\pi_{A_{1}}^{t}-\pi_{S_{-1}}^{* t}\right)$ and, by definition, is larger than the payoff from any other feasible combination of journals in $Q$. We prove that for a large $t$ this leads to a contradiction. We start by observing that, as $t \rightarrow+\infty, \mathbf{p}_{-1}^{* t}$ tends to $\mathbf{p}_{u}^{n-n_{1}} \equiv\left(p^{*}, \ldots, p^{*}\right) \in \mathbb{R}^{n-n_{1}}$, where $p^{*}$ is determined by Proposition 1 (this fact is both intuitive and simple to prove). Then, we can show that publisher 1 makes all his journals active in any profitable deviation and $\lim _{t \rightarrow+\infty} \mathbf{p}_{1}^{t}=$

\footnotetext{
${ }^{39}$ This fact is obvious if the industry profit $\pi^{* t}$ before $j(t)$ 's deviation is equal to $M$. If instead $\pi^{* t}<M$, then $u_{i j}^{t}=U_{M B}\left(p_{i j}^{* t}, \pi^{* t}\right)$ for any $i j$ with $j \neq j(t)$. In the case that $j(t)$ 's profit increases by $\varepsilon>0$ and all journals of the other publishers are purchased, the industry profit increases to $\pi^{* t}+\varepsilon>\pi^{* t}$. But $u_{i j}^{t} \geq U_{M B}\left(p_{i j}^{* t}, \pi^{* t}+\varepsilon\right)$ cannot hold for any $i j$ with $j \neq j(t)$ and lemma 2 implies that the library does not buy all journals of publishers different from $j(t)$.
} 
$\mathbf{p}_{u}^{n_{1}} \equiv\left(p^{*}, \ldots, p^{*}\right) \in \mathbb{R}^{n_{1}}$.

Claim $1 A_{1}=\left\{11,21, \ldots, n_{1} 1\right\}$ and $\lim _{t \rightarrow+\infty} \mathbf{p}_{1}^{t}=\mathbf{p}_{u}^{n_{1}}$.

Proof First notice that a subsequence of $\mathbf{p}_{1}^{t}$ converges; without loss of generality, we suppose that the subsequence is the original sequence; let $\mathbf{p}_{1}$ denote $\lim _{t \rightarrow+\infty} \mathbf{p}_{1}^{t}$. Since $\left(A_{1}, \mathbf{p}_{1}^{t}\right)$ is a profitable deviation for 1 , the inequality $\pi_{A_{1}}^{t}>\sum_{i=1}^{n_{1}} p_{i 1}^{* t}$ holds for any $t$. Furthermore, $\lim _{t \rightarrow+\infty} \sum_{i=1}^{n_{1}} p_{i 1}^{* t}=n_{1} p^{*}$ and therefore

$$
\lim _{t \rightarrow+\infty} \pi_{A_{1}}^{t}=\sum_{i 1 \in A_{1}} p_{i 1} \geq n_{1} p^{*}
$$

Now assume by contradiction that $\mathbf{p}_{1} \neq \mathbf{p}_{u}^{\left|A_{1}\right|}$. Notice that $\mathbf{p}_{1} \neq \mathbf{p}_{u}^{\left|A_{1}\right|}$ implies $p_{i 1}>p^{*}$ for at least one $i 1 \in A_{1}$ since otherwise $p_{i 1} \leq p^{*}$ for any $i 1 \in A_{1}$ and this together with $\mathbf{p}_{1} \neq \mathbf{p}_{u}^{\left|A_{1}\right|}$ violates (28). Without loss of generality, we suppose that $11 \in A_{1}$ and $p_{11}>p^{*}$. Since $S_{-1}$ is a strict subset of $\bar{S}_{-1}$, let $i^{\prime} j^{\prime} \in\left(\bar{S}_{-1} \backslash S_{1}\right) \neq \emptyset$ be a journal of publisher $j^{\prime}$ with $j^{\prime} \neq 1$ which the library does not buy given $\left(A_{j(t)}^{t}, \mathbf{p}^{t}\right)$. Since $\lim _{t \rightarrow+\infty} p_{i^{\prime} j^{\prime}}^{* t}=$ $p^{*}$ and $\lim _{t \rightarrow+\infty} p_{11}^{t}=p_{11}>p^{*}$, it is obvious that $\left(A_{1} \backslash\{11\} \cup S_{-1} \cup\left\{i^{\prime} j^{\prime}\right\}\right) \in Q$ for any large $t$. The library's payoff from buying the journals in $A_{1} \backslash\{11\} \cup S_{-1} \cup\left\{i^{\prime} j^{\prime}\right\}$ is $U_{A_{1}}^{t}-u_{11}^{t}+U_{S_{-1}}^{t}+u_{i^{\prime} j^{\prime}}^{t}+v\left(M-\pi_{A_{1}}^{t}+p_{11}^{t}-\pi_{S_{-1}}^{* t}-p_{i^{\prime} j^{\prime}}^{* t}\right)$ and for any large $t$ this is larger than the payoff from buying the journals in $A_{1} \cup S_{-1}$ : a contradiction. Since $p_{i 1} \leq p^{*}$ for any $i 1 \in A_{1},(28)$ implies $A_{1}=\left\{11,21, \ldots, n_{1} 1\right\}$ and $p_{i 1}=p^{*}$ for $i=1, . ., n_{1} . \diamond$

Claim 1 says that (for a large $t$ ), any profitable deviation of publisher 1 is such that all of his journals are active, the library buys all of them and the price vector $\mathbf{p}_{1}^{t}$ is close to $\mathbf{p}_{u}^{n_{1}}$; hence, $\mathbf{p}_{1}^{t}$ is also close to $\mathbf{p}_{1}^{* t}$. Next claim establishes a result about the journals of the other publishers the library buys given 1's deviation.

Claim 2 The set $\bar{S}_{-1} \backslash S_{-1}$ includes only one journal.

Proof Since $\lim _{t \rightarrow+\infty} \mathbf{p}_{1}^{t}=\mathbf{p}_{u}^{n_{1}}, \sum_{i=1}^{n_{1}} p_{i 1}^{t}+\sum_{i j \in \bar{S}_{-1}} p_{i j}^{* t}$ is only slightly larger than $\sum_{i=1}^{n_{1}} p_{i 1}^{* t}+$ $\sum_{i j \in \bar{S}_{-1}} p_{i j}^{* t}$ for a large $t$ and therefore $\sum_{i=1}^{n_{1}} p_{i 1}^{t}+\sum_{i j \in\left(\bar{S}_{-1} \backslash i^{\prime} j^{\prime}\right)} p_{i j}^{* t}<\sum_{i=1}^{n_{1}} p_{i 1}^{* t}+\sum_{i j \in \bar{S}_{-1}} p_{i j}^{* t}$ if $i^{\prime} j^{\prime}$ is an arbitrary journal of a publisher $j^{\prime}$ with $j^{\prime} \neq 1$. Furthermore, recall that $\mathbf{p}^{* t}$ is such that $u_{i j}^{t} \geq U_{M B}\left(p_{i j}^{* t}, \sum_{i=1}^{n_{1}} p_{i 1}^{* t}+\sum_{i j \in \bar{S}_{-1}} p_{i j}^{* t}\right)$ for any $i j$. Hence, $u_{i j}^{t}>U_{M B}\left(p_{i j}^{* t}, \sum_{i=1}^{n_{1}} p_{i 1}^{t}+\right.$ $\left.\sum_{i j \in\left(\bar{S}_{-1} \backslash i^{\prime} j^{\prime}\right)} p_{i j}^{* t}\right)$ for any $i j \neq i^{\prime} j^{\prime}$ with $j \neq 1$. Since the library buys all journals of 1 , this means (by lemma 2) that it will drop exactly one journal of the other publishers. $\diamond$

Claims 1 and 2 imply that for a large $t$, at prices $\mathbf{p}^{t}$ the library buys all the journals of publisher 1 and all but one journal of the other publishers. However, next claim shows that this is impossible, given approximate homogeneity.

Claim 3 If journals are almost homogeneous, no price vector for the journals of 1 induces the library to drop exactly one journal of the other publishers while buying all journals of 1 . 
Proof Let publisher 1 modify the prices of journals 11 to $n_{1} 1$ by $\varepsilon_{1}, \ldots, \varepsilon_{n_{1}}$, respectively, with $\varepsilon=\varepsilon_{1}+\ldots+\varepsilon_{n_{1}}>0 ;{ }^{40}$ the new prices for journals of 1 are $p_{i 1}=p_{i 1}^{*}+\varepsilon_{i}, i=1, \ldots, n_{1}$. The library's payoff if it drops a journal $i j(j \neq 1)$ is $U-u_{i j}+v\left(M-\pi+p_{i j}^{*}-\varepsilon\right)$, reduced by $v\left(M-\pi+p_{i j}^{*}\right)-v\left(M-\pi+p_{i j}^{*}-\varepsilon\right)+b(b=0$ if $\pi<M$ and $b \geq 0$ if $\pi=M)$ with respect to the payoff $U+v(M-\pi)$ before the change in prices by 1 . Since $v$ is strictly concave, this reduction in payoff is minimized for the journal $i j(j \neq 1)$ with the highest price, which we denote by $\bar{p}_{-1}^{*}$. If instead the library does not buy a journal $i 1$, its payoff is $U-u_{i 1}+v\left(M-\pi+p_{i 1}^{*}-\varepsilon+\varepsilon_{i}\right)$, reduced by $v\left(M-\pi+p_{i 1}^{*}\right)-v\left(M-\pi+p_{i 1}^{*}-\varepsilon+\varepsilon_{i}\right)+b$ with respect to $U+v(M-\pi)$. Therefore, the library prefers to drop the highest priced journal of the other publishers rather than one journal of 1 if $v\left(M-\pi+\bar{p}_{-1}^{*}\right)-v\left(M-\pi+\bar{p}_{-1}^{*}-\varepsilon\right)<$ $v\left(M-\pi+p_{i 1}^{*}\right)-v\left(M-\pi+p_{i 1}^{*}-\varepsilon+\varepsilon_{i}\right)$ for $i=1, \ldots, n_{1}$; this set of conditions is equivalent to $v\left(M-\pi+\bar{p}_{-1}^{*}\right)-v\left(M-\pi+\bar{p}_{-1}^{*}-\varepsilon\right)<\phi\left(\varepsilon_{1}, \ldots, \varepsilon_{n_{1}}\right) \equiv \min \left\{v\left(M-\pi+p_{i 1}^{*}\right)-v(M-\right.$ $\left.\left.\pi+p_{i 1}^{*}-\varepsilon+\varepsilon_{i}\right), i=1, \ldots, n_{1}\right\} .{ }^{41}$ This shows that, given $\varepsilon$, publisher 1 is interested in choosing $\varepsilon_{1}, \ldots, \varepsilon_{n_{1}}$ which maximize $\phi$. The optimal values of $\varepsilon_{1}, \ldots, \varepsilon_{n_{1}}$ are denoted by $\bar{\varepsilon}_{1}, \ldots, \bar{\varepsilon}_{n_{1}}$ and satisfy

$$
\begin{gathered}
v\left(M-\pi+p_{i 1}^{*}\right)-v\left(M-\pi+p_{i 1}^{*}-\varepsilon+\varepsilon_{i}\right)=v\left(M-\pi+p_{n_{1} 1}^{*}\right)-v\left(M-\pi+p_{n_{1} 1}^{*}-\varepsilon+\varepsilon_{n_{1}}\right) \\
\text { for } i=1, \ldots, n_{1}-1
\end{gathered}
$$$$
\varepsilon_{1}+\varepsilon_{2}+\ldots+\varepsilon_{n_{1}}=\varepsilon
$$

provided that $M-\pi+p_{i 1}^{*}-\varepsilon+\bar{\varepsilon}_{i}>0$ for $i=1, \ldots, n_{1}$. If this is the case, then $\phi\left(\bar{\varepsilon}_{1}, \ldots, \bar{\varepsilon}_{n_{1}}\right)=$ $v\left(M-\pi+p_{11}^{*}\right)-v\left(M-\pi+p_{11}^{*}-\varepsilon+\bar{\varepsilon}_{1}\right)$; otherwise, $\phi\left(\bar{\varepsilon}_{1}, \ldots, \bar{\varepsilon}_{n_{1}}\right) \leq v\left(M-\pi+p_{11}^{*}\right)-$ $v\left(M-\pi+p_{11}^{*}-\varepsilon+\bar{\varepsilon}_{1}\right)$. We prove below that

$$
v\left(M-\pi+\bar{p}_{-1}^{*}\right)-v\left(M-\pi+\bar{p}_{-1}^{*}-\varepsilon\right)>\phi\left(\bar{\varepsilon}_{1}, \ldots, \bar{\varepsilon}_{n_{1}}\right)
$$

when $M-\pi+p_{i 1}^{*}-\varepsilon+\bar{\varepsilon}_{i}>0$ for $i=1, \ldots, n_{1}$; hence, we conclude that (30) holds a fortiori $M-\pi+p_{i 1}^{*}-\varepsilon+\bar{\varepsilon}_{i}=0$ for some $i$. Given that journals are approximately homogeneous, $p_{i 1}^{*}$ is close to $p_{n_{1} 1}^{*}$ for $i=1, \ldots, n_{1}-1$ and therefore (29) implies that $\bar{\varepsilon}_{i}$ is close to $\bar{\varepsilon}_{n_{1}}$, or $\bar{\varepsilon}_{i}$ is close to $\frac{\varepsilon}{n_{1}}>0$ for $i=1, \ldots, n_{1}$. Then (30) holds because $\bar{p}_{-1}^{*}$ is close to $p_{11}^{*}$ and the left hand side of (30) is close to $v\left(M-\pi+p_{11}^{*}\right)-v\left(M-\pi+p_{11}^{*}-\varepsilon\right)$, while the right hand side is close to $v\left(M-\pi+p_{11}^{*}\right)-v\left(M-\pi+p_{11}^{*}-\varepsilon+\frac{\varepsilon}{n_{1}}\right)$.

\footnotetext{
${ }^{40}$ Notice that we allow that the prices of some journals of 1 are not changed.

${ }^{41}$ With $n_{1}=1$ this inequality fails to hold because $\varepsilon_{1}=\varepsilon$ and the right hand side is 0 .
} 